\title{
Nonlinear Acoustic Metamaterials for Sound Attenuation Applications
}

Final Report, DARPA STO Seedling

Jinkyu Yang and Chiara Daraio (PI)

Engineering and Applied Science, California Institute of Technology

1200 E. California Blvd., MC 105-50, Pasadena, California 91125, USA

\section{Sponsored by}

Defense Advanced Research Projects Agency

Strategic Technology Office/STO

Program: Nonlinear Acoustic Metamaterials

Dr. Jinendra Ranka

Issued by DARPA/CMO under Contract No. HROO $11-10-\mathrm{C}-0089$

\section{Disclaimer}

The views and conclusions contained in this document are those of the authors and should not be interpreted as representing the official policies, either expressly or implied, of the Defense Advanced Research Projects Agency or the U.S. Government.

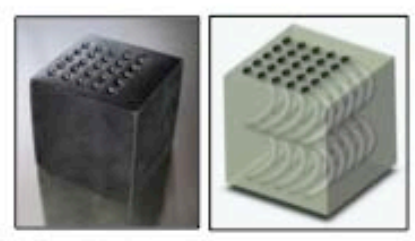

- Novel material systems

- Tunable acoustic filtering structures

- Wave dynamics in complex systems: geometry and contact

- Interaction between linear and nonlinear media

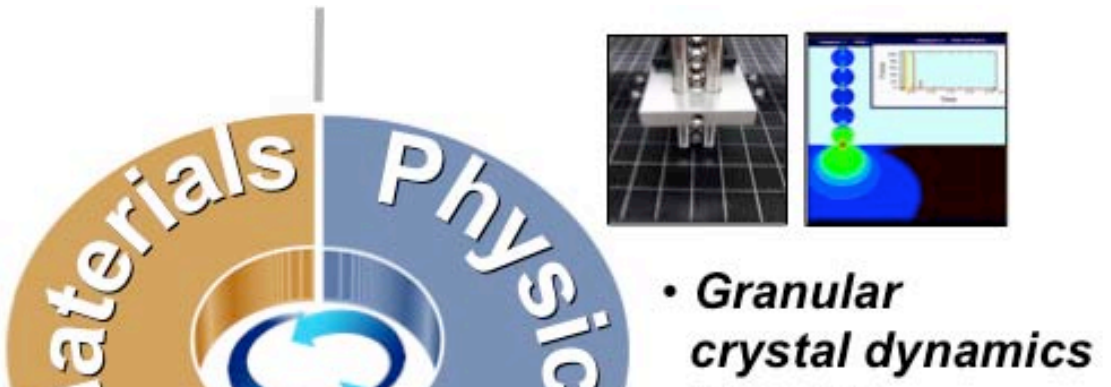

- Tunable nonlinear waves

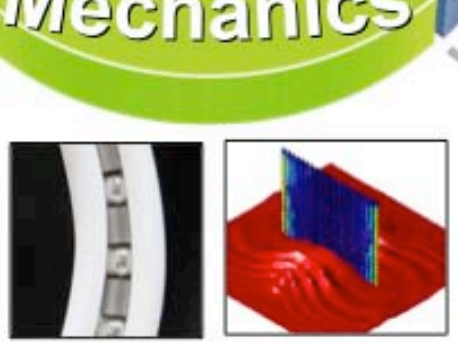




\section{Report Documentation Page}

Form Approved

OMB No. 0704-0188

Public reporting burden for the collection of information is estimated to average 1 hour per response, including the time for reviewing instructions, searching existing data sources, gathering and maintaining the data needed, and completing and reviewing the collection of information. Send comments regarding this burden estimate or any other aspect of this collection of information,

including suggestions for reducing this burden, to Washington Headquarters Services, Directorate for Information Operations and Reports, 1215 Jefferson Davis Highway, Suite 1204, Arlington

VA 22202-4302. Respondents should be aware that notwithstanding any other provision of law, no person shall be subject to a penalty for failing to comply with a collection of information if it

does not display a currently valid OMB control number.

\begin{tabular}{|c|c|c|}
\hline $\begin{array}{l}\text { 1. REPORT DATE } \\
\mathbf{1 6} \text { MAR } 2011\end{array}$ & 2. REPORT TYPE & $\begin{array}{l}\text { 3. DATES COVERED } \\
\mathbf{0 0 - 0 0 - 2 0 1 1} \text { to } 00-\mathbf{0 0 - 2 0 1 1}\end{array}$ \\
\hline \multirow{3}{*}{\multicolumn{2}{|c|}{$\begin{array}{l}\text { 4. TITLE AND SUBTITLE } \\
\text { Nonlinear Acoustic Metamaterials For Sound Attenuation Applications }\end{array}$}} & 5a. CONTRACT NUMBER \\
\hline & & 5b. GRANT NUMBER \\
\hline & & 5c. PROGRAM ELEMENT NUMBER \\
\hline \multirow{3}{*}{\multicolumn{2}{|c|}{ 6. AUTHOR(S) }} & 5d. PROJECT NUMBER \\
\hline & & 5e. TASK NUMBER \\
\hline & & 5f. WORK UNIT NUMBER \\
\hline \multicolumn{2}{|c|}{$\begin{array}{l}\text { 7. PERFORMING ORGANIZATION NAME(S) AND ADDRESS(ES) } \\
\text { California Institute of Technology,Engineering and Applied Science,1200 } \\
\text { E California Blvd,Pasadena,CA,91125 }\end{array}$} & $\begin{array}{l}\text { 8. PERFORMING ORGANIZATION } \\
\text { REPORT NUMBER }\end{array}$ \\
\hline \multirow{2}{*}{\multicolumn{2}{|c|}{ 9. SPONSORING/MONITORING AGENCY NAME(S) AND ADDRESS(ES) }} & 10. SPONSOR/MONITOR'S ACRONYM(S) \\
\hline & & $\begin{array}{l}\text { 11. SPONSOR/MONITOR'S REPORT } \\
\text { NUMBER(S) }\end{array}$ \\
\hline
\end{tabular}

12. DISTRIBUTION/AVAILABILITY STATEMENT

Approved for public release; distribution unlimited

13. SUPPLEMENTARY NOTES

14. ABSTRACT

We design and test nonlinear acoustic materials for amplitude dependent filtering of audible signals. We study the dynamic response of one-dimensional arrays of elastic particles in contact with each other, supported by deformable elastic rods, using experiments and numerical simulations. We study the effects of different curvatures of the support rods on the transmission of impacts and structural vibrations. We find that the combination of a nonlinear granular medium with linear support rods allows obtaining tunable filtering of the incoming signal, and selecting desired ranges of the transmitted frequency and amplitude. We conclude our study building a 3D prototype of the new filtering material using a 3D printer.

15. SUBJECT TERMS

16. SECURITY CLASSIFICATION OF:

a. REPORT

unclassified b. ABSTRACT unclassified c. THIS PAGE unclassified
17. LIMITATION OF ABSTRACT

Same as Report (SAR)
18. NUMBER 19a. NAME OF

OF PAGES RESPONSIBLE PERSON

27 


\section{TASK OBJECTIVES}

The overall task objective is to develop a concept demonstrator of a novel acoustic filter system that transmits or mitigates external impacts in a selected range of frequencies. Furthermore, the new isolator is to be equipped with amplitude-dependent acoustic filtering capability, allowing highly efficient transmission of small dynamic disturbances relative to that of strong excitations. This material will be useful in engineering applications, such as protecting soldiers against hearing damage from weapons fire while largely retaining situational awareness.

The research component related to this effort is aimed at creating and understanding new highly nonlinear acoustic metamaterials based on "engineered", ordered granular crystals. Granular crystals are highly ordered aggregates of particles in elastic contact with each other, and they exhibit extremely versatile dynamic responses encompassing linear to highly nonlinear acoustic regimes. Leveraging the added degree of freedom in controlling wave dynamics in granular crystals, we plan to adjust the targeted ranges of filtering frequencies in a controllable manner. The range of allowable and forbidden frequency bands will be within the limit of audible frequencies $(20 \sim 20,000 \mathrm{~Hz})$. The amplitude-dependent attenuation of external impacts will be achieved by developing a new energy absorption mechanism based on the architecture of combined granular crystals and soft linear elastic media. This hybrid system will not rely on any onset of permanent deformation of granules or structural buckling of their containment.

For the proof-of-concept, we will focus on the development of one-dimensional prototype. The verification of its feasibility will be demonstrated by both experiments and numerical simulations. Based on the findings in one-dimensional study, we suggest a design guideline for potential fabrication of threedimensional structures in future follow-up studies.

\section{I.1 TECHNICAL APPROACH}

An acoustic crystal composed of tightly packed spherical particles can exhibit a wide spectrum of acoustic properties with responses varying from linear to highly nonlinear regimes. The physical attractiveness of these crystals resides in the controllability of such acoustic responses by simple manipulation of static pre-compression applied to the material. During the first three months of this study we focused on the fundamental understanding of the energy transmission through these crystals in relation to the tunable acoustic nonlinearity. To control the degree of nonlinearity, we varied three parameters, i) pre-compression, ii) striker velocity and iii) striker mass, maintaining an identical configuration of one-dimensional (1D) granular structures. We experimentally and numerically evaluated the transmission gain in the stop/pass frequency band of the granular chain as a function of the nonlinearity (i.e. as a function of the precompression applied). We also studied the evolution of the frequency band structure as the degree of nonlinearity was changed. We found that the transmission gain of the granular structure shows a remarkable dependence on the structural linearity level.

To combine the frequency filtering response governed by the discrete particles with an amplitude filtering response, we assembled a system composed of a highly nonlinear granular chain and a deformable linear medium. We found that the acoustic wave propagation can be efficiently manipulated and redirected with such added degree of freedom. During the second trimester, we built and tested a hybrid linear/nonlinear metamaterial to allow high-energy transmission only in a selected range of external impact amplitude. In this hybrid structure, the nonlinear granular chain takes the role of transmitting energy when the system is excited with low amplitude impacts, whereas the linear structure performs as an effective shock mitigation medium under large amplitude impacts, controlled by structural deflections. We experimentally verified a strong correlation of transmission gain with external impact amplitudes, showing an order-of-magnitude reduction of transmission gain for large amplitude impacts compared to that of low amplitude impacts. We numerically examined the wave propagation and impact mitigation in the nonlinear acoustic metamaterial using a combined discrete particle model and a finite element method. Finally, we verified that the numerical results are in excellent agreement with the experimental results. 
During the third trimester we studied the hybrid linear/nonlinear system under continuous structural vibrations and noise. We tested systems composed of uniform particles and layered systems composed of alternating particles. This allowed us to study the fundamental effects of tunable dispersion relations and band gaps with the amplitude dependent filtering. To validate our findings we performed numerical simulations finding good agreement.

During the final trimester we began preparing publications of our findings and extended the design of our materials from 1-D to 3-D. We proposed the use of multi-material 3-D printing to obtain the desired complex structures capable of combining the linear and nonlinear responses. We printed a prototype as a final accomplishment of this project.

\section{TECHNICAL PROBLEMS}

In this study, we designed tunable acoustic metamaterials based on strongly nonlinear wave dynamics. The proposed metamaterial are fundamentally different from any other approach to vibration isolation. They do not use active modulation to suppress external vibration/impacts but rely on passive insulation. Furthermore, they are stiff and load bearing, present large recovery to external deformation and do not develop permanent damage in the ranges of excitations studied. The proposed systems are designed to forbid the propagation of waves in selected frequency ranges (also called as band gaps or stop bands). Incident waves in these forbidden frequency ranges experience an exponential decay of their amplitudes (i.e., they are evanescent waves), and they are fully reflected. The presence of nonlinearity in the structure may allow the redirection of part of the incoming energy into allowed modes.

To construct this new type of isolator using highly nonlinear acoustic metamaterials, several key technical problems exist as listed below:

\section{(1) Experimental observation of the presence of tunable band-gaps}

The creation of tunable frequency band-gaps is the first technical challenge in this study. To observe the presence of band-gaps, we studied the dispersion relation in granular crystals, and investigated the sound transmissibility targeting selected frequency bands in audible frequency ranges. Based on this fundamental understanding, we tuned the design of the new material systems controlling their geometry and structural configurations.

\section{(2) Experimental verification of amplitude-dependent attenuation of acoustic signals}

One of the unique physical properties of highly nonlinear dynamic systems is that the wave speed in the media is dependent on the signal amplitude. However, the dependence of signal attenuation on its amplitude has not been fully investigated in previous studies. In this study, we examined the wave attenuation, thus energy transmission efficiency, through the granular crystals over a range of nonlinear waves' amplitudes. We observed a significant effect of the waves' amplitudes on their attenuation, and exploited this property to construct the amplitude-dependent filtering systems. To provide an enhanced efficiency in absorbing external energy under larger-amplitude impacts, we employed soft linear media that surrounds and couples with the nonlinear granular arrays.

\section{(3) Numerical modeling of the wave dynamics in nonlinear acoustic metamaterials}

Previous studies have shown that wave dynamics in one-dimensional granular crystals can be successfully modeled using a discrete element (DE) model. In this study, we developed an improved version of the classical discrete particle model that allowed accounting for the interaction of nonlinear metamaterials with their surrounding linear media. Modeling of the wave dynamics in the coupled linear and nonlinear media was challenging due to the interplay between granular particles and soft elastic media. To resolve this technical problem, integrated the DE model into a finite element (FE) code. 


\section{GENERAL METHODOLOGY}

\section{III-1. Background}

Chains of granular particles in elastic contact with each other (also defined as one-dimensional granular crystals) have been broadly studied to control compressive excitations [1-23]. Given external loads, such as impulse and harmonic excitations, these systems can be tuned to respond in different acoustic regimes, ranging from linear, weakly nonlinear, to highly nonlinear states [1]. It has been shown that the alteration of the dynamic regime can be easily made by manipulating precompression initially applied to the granular crystals [1,6]. Under strong precompression, these closely packed granular crystals exhibit acoustic band gaps with distinctive pass and forbidden frequency bands, resulting from linear dispersion [4, 7]. In the case of zero or very weak precompression, one of the most studied characteristics of granular crystals is the ability to support the formation and propagation of highly nonlinear acoustic waves in the form of solitary waves [1,2]. These solitary waves are compactlysupported energy lumps, that derive from a balance between dispersive and nonlinear effects in the chain of elastic particles. They are characterized by unique physical properties, such as robustness, amplitudedependent wave propagation speed, and high energy intensity $[1,8,9]$.

Solitary waves propagating in granular crystals have been proposed for several engineering applications, such as nonlinear lenses for acoustic imaging [9], sound scramblers [10], and actuators and sensors for nondestructive evaluations [11, 12]. In particular, previous studies have suggested that nonlinear granular crystals can act as effective media for shock/impact mitigation, owing to their ability to trap [3, 11], localize [13, 24], redirect [14, 15], and redistribute energy [16]. These capabilities are based on the complex dynamic behavior of granular chains composed of particles of different materials and geometry. The presence of dissipation, evident in experimental studies of granular crystals, provides the additional means for impact mitigation. Several reports have recently provided different theoretical models to capture the force decay observed in experiments [17-19].

In this project we study the dynamic response of a curved chain of uniform and diatomic "spherecylinder" particles constrained by structurally deformable elastic guides. We excite compressive waves in this system with two types of axial loading: small-amplitude disturbances generated by a piezoelectric actuator and large-amplitude impacts by different falling masses. We also vary the chain's initial curvature imposed by the elastic guides to study the attenuation effects related to the structural arrangements of granules. This combined system of granular particles and linear support media allows studying the effects of structural bending of the elastic supports on the propagation of nonlinear waves in the granular chains.

\section{ACTIVITIES AND RESULTS}

\section{Experimental studies on transient responses of 1D straight chain}

\section{Experimental Setup}

The dynamic responses of a 1D phononic crystal were investigated under various nonlinearity conditions. We assembled a granular crystal composed of 20 stainless steel beads (McMaster, 440C, diameter 3/4"), which were guided by four vertical rails [Fig. 1]. The granular chain was impacted by a cylindrical striker with various masses and drop heights to impose a wide range of dynamic disturbances. The pre-compression of the $1 \mathrm{D}$ chain was also manipulated by adding different weights on the top of the chain for the purpose of controlling the chain stiffness level.

In the $1 \mathrm{D}$ granular chain, previous studies have shown that a higher degree of nonlinearity is obtained by increasing $F_{\mathrm{d}} / F_{0}$, the ratio of dynamic disturbance over the pre-compressive force [1-2]. In this study, therefore, we enhanced the nonlinearity level of the 1D chain by i) increasing striker mass, ii) increasing striker velocity, or iii) reducing pre-compression. The transmitted force was measured by a commercial force sensor (PCB C01 C05) at the bottom of the chain, which is connected to a data acquisition board (NI PCI-6115) to collect experimental data. 


\section{Experimental Results}

As a higher degree of system nonlinearity is imposed on the granular chain, it starts to form highly nonlinear solitary waves under a striker impact, which is an unprecedented way of wave propagation never before observed in inear systems [3]. Figure 2 reports the smooth transition of wave configurations from linear (top grey curve) to highly nonlinear ones (bottom blue curve), as the system nonlinearity is increased due to the reduction of static pre-compression. We observe that the solitary wave exhibits a nondispersive, compactly-supported shape with an order-of-magnitude higher force amplitude than that of the linear wave under the identical impact condition. Extremely slow propagation speed and fixed wave's width (five particle diameters in a homogeneous spherical chain) are other unique characteristics of the solitary waves [2]. On the other hand, a force profile measured under the linear status of the chain shows a dispersive and oscillatory wave propagation trend, which is similar to that of harmonic waves in continuum media.

This experimental observation reveals that the 1D granular chain under the nonlinear condition is an efficient medium for transmitting energy in the form of the highly nonlinear solitary waves. On the contrary, the identical granular chain becomes better suited for the purpose of external impact mitigations, if it is converted to the linear configuration using higher pre-compression. In this case, the reduction of transmitted energy can be explained by dissipation mechanism, such as friction among particles and waves' dispersion in the linear chain. Further experimental results will be discussed in the later section.

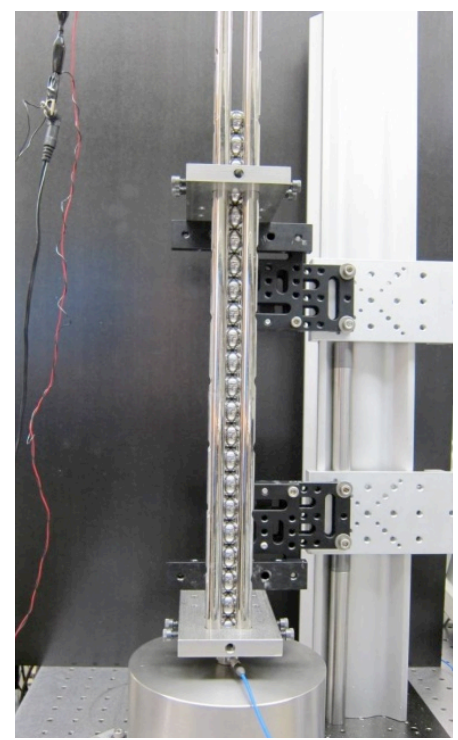

Figure 1. 1D straight chain composed of 20 stainless steel beads. PCB force sensor is located at the bottom of the chain.

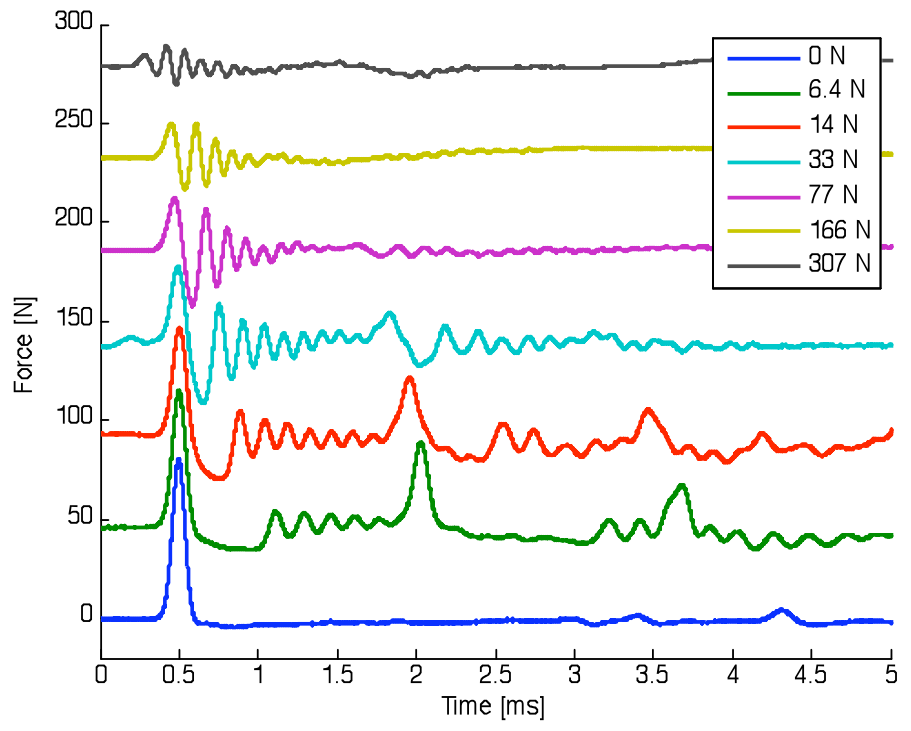

Figure 2. Experimental force profiles of transmitted waves through the monomer chain of spherical particles under various pre-compressions on the chain. The force profile positioned at the top (gray line) shows an oscillatory harmonic wave under high pre-compression, whereas the bottom curve (blue line) corresponds to a compactly-supported solitary wave under no pre-compression.

\section{Numerical studies on transient responses of 1D straight chain}

\section{Numerical model}

The particle interactions in this 1D straight chain are regulated by Hertzian contact law expressed as $F \propto \delta^{3 / 2}$ where $F$ is force and $\delta$ is approach between particles. The equation of motion of such a nonlinear spring-type spherical lattice in $1 \mathrm{D}$ alignment can be expressed as: 


$$
\begin{gathered}
m \ddot{u}_{n}=A\left[u_{n-1}-u_{n}\right]_{+}^{3 / 2}-A\left[u_{n}-u_{n+1}\right]_{+}^{3 / 2} n \in\{1, \ldots, N-1\} \\
A=E \sqrt{2 R} / 3\left(1-v^{2}\right) .
\end{gathered}
$$

Here $m$ is the mass of the bead, and $u_{n}$ is the displacement of the given particle from its equilibrium position [4]. The coefficient of the nonlinear interaction $A$ is a function of $E$, $v$, and $R$, which are Young's modulus, Poisson's ratio, and the radius of the granular element. The bracket $[s]_{+}$takes only positive values and returns 0 if $\mathrm{s}<0$. We numerically investigated the generation and propagation of the solitary waves, as well as harmonic waves, in the 1D granular chain by solving this equation with the aid of the ordinary differential equation solvers in MATLAB [5].

\section{Comparison of experimental and numerical results}

We experimentally and numerically evaluated the acoustic filtering behaviors of the granular chain under various conditions of nonlinearity. To form a frequency band structure, we used a diatomic chain composed of alternating 3/4"-diameter beads and 3/4"-height cylinders. In this case, we expect to generate two band passes, acoustic and optical bands, and a band gap between the two passes [1]. We can analytically obtain a lower and upper bound of the band gap expressed by the following equations [1]:

$$
f_{1}=\frac{1}{2 \pi}\left(\frac{2 \beta}{m_{1}}\right)^{1 / 2}, \quad f_{2}=\frac{1}{2 \pi}\left(\frac{2 \beta}{m_{2}}\right)^{1 / 2} .
$$

Here $m_{1}$ and $m_{2}$ are masses of cylinder and bead, respectively, and $\beta$ is a constant proportional to the materials parameters (Young's modulus, Poisson's ratio, and particle's radii) and pre-compression applied to the system. This means that the locations of the frequency band structure can be altered by the choice of the material and the static pressure on the granular crystal.

The experimental and numerical frequency band structures are reported in Fig. 3, using three different methods of nonlinearity manipulation: i) pre-compression, ii) striker velocity, and iii) striker mass. It is notable that unlike two other cases, the change of pre-compression alters the locations of pass/stop bands, as shown in Figs. 3 (a) and (b). This is in accordance with the analytic prediction by Eq. (2). From all three groups of results, regardless of the methodology, we can observe that the band structure becomes less distinctive as the degree of nonlinearity increases. This is because the dispersion phenomenon that forms frequency band structures is a characteristic property of linear systems. Another important finding in experiment is that the transmission gain in the pass band, as well as in the stop band, increases as the degree of nonlinearity rises [see the increase of color intensity towards the right of the graphs in Fig. 3]. This is due to the reduced amount of energy dissipation, caused by less friction and more efficient nonlinear wave propagation, under the nonlinear configuration of the diatomic granular crystal. In the simulation results where the friction effect is not taken into account, the discrepancy of energy dissipation between the linear and nonlinear systems is not as obvious as in the experimental results.

\section{Experimental studies on harmonic responses of 1D straight chain}

To observe the effect of excitation amplitudes on the formation of the frequency band structure, we applied harmonic perturbations, instead of transient striker impact, to the 1D diatomic chain with 2:1 periodicity, by means of piezo-actuator and electrically controlled amplifier unit. In this way, we can precisely manipulate the excitation amplitudes in a broadband frequency domain. The experimental results are reported in Fig. 4. The pass and stop frequency bands are clearly observable in all the range of amplifier gains considered. However, despite the twentyfold gain amplification, we observe a negligible shift of pass/stop band frequencies. Previous studies also reported insignificant dependence of dispersion relationship on the excitation amplitudes [6]. For a more dramatic change of the frequency band structure, we need to develop a complex system that exploits the nonlinear properties of granular crystals 
(a) Experiments: Pre-compression

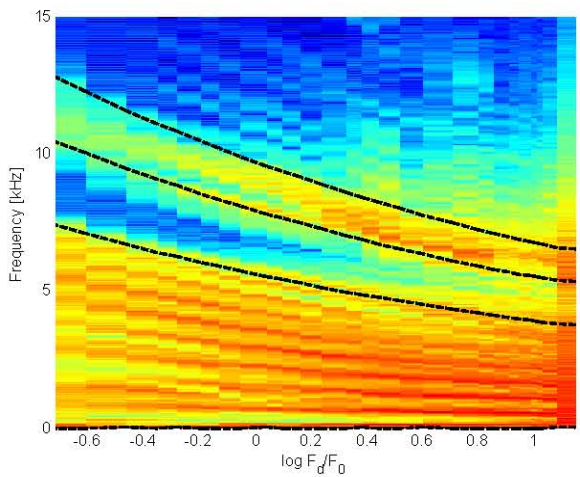

(c) Experiments: Striker velocity

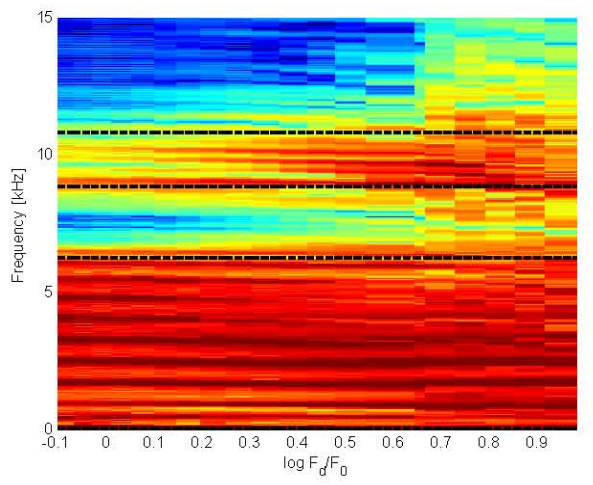

(e) Experiments: Striker mass

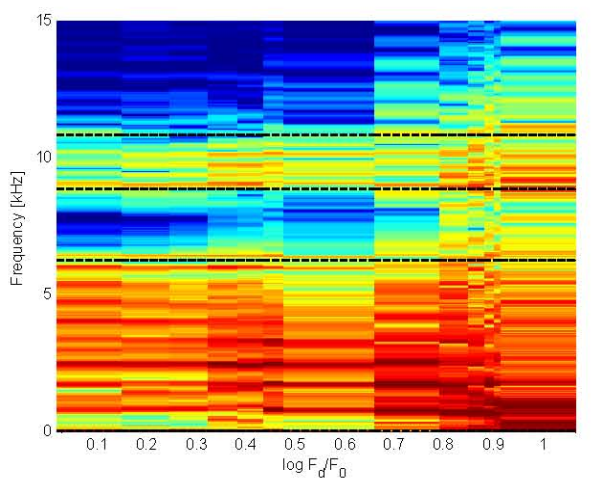

(b) Simulations: Pre-compression

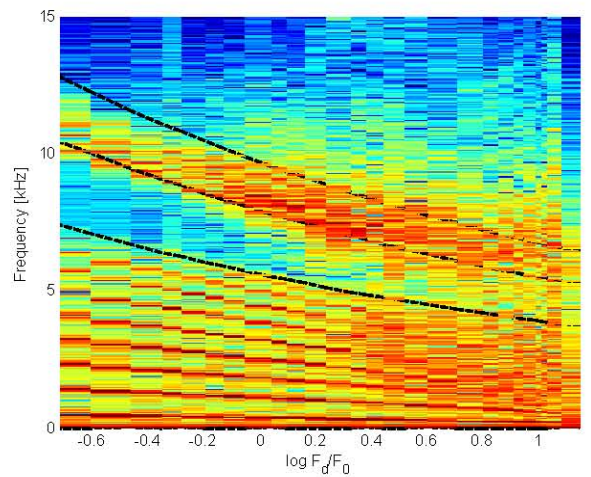

(d) Simulations: Striker velocity

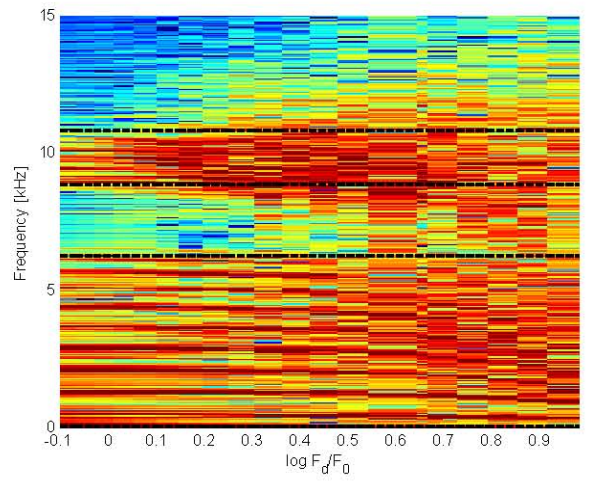

(f) Simulations: Striker mass

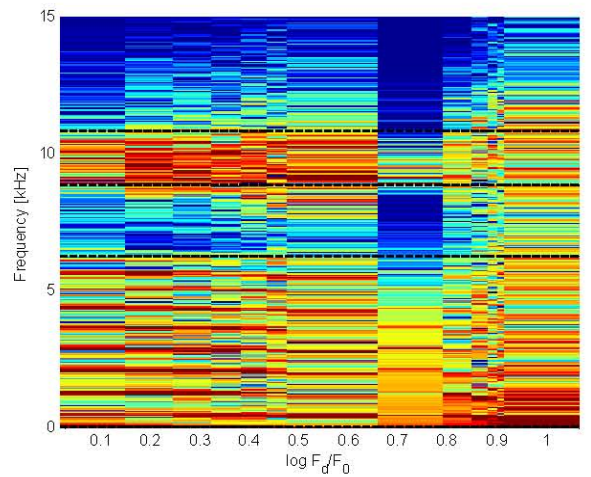

Figure 3. Experimental and numerical results of a diatomic band structure as a function of imposed nonlinearity. The nonlinearity condition was varied by pre-compression [(a) and (b)], striker velocity [(c) and (d)], and striker mass [(e) and (f)]. The degree of nonlinearity increases as the $F_{\mathrm{d}} / F_{0}$ value increases. The red-colored zone represents band pass, while blue-colored zone corresponds to band gap. Black dotted lines denote cutoff frequencies based on theoretical dispersion relationship.

\section{Experimental studies on transient responses of 1D curved chain}

\section{Experimental Setup}

We built a 1D curved chain of hybrid linear/nonlinear structure to exploit its versatile energy transmission capability under various impact conditions [Fig. 5]. The curved chain is composed of 20 particles of stainless steel beads - identical to the ones used in the previous straight chain - which are guided by 4 pre-curved rods made of soft polytetrafluoroethylene (PTFE) material. The rods are held together by two steel plates, and the top plate has both translational (in vertical direction) and rotational degrees of freedom. This allows structural bending motion of the hybrid structure under the striker impact 
applied to the top bead of the granular chain. Given small amplitude of striker impact, the propagating energy is mainly carried by the highly nonlinear granular chain in the form of solitary waves, facilitating efficient transmission of impact energy. When the hybrid structure strongly collides with a heavy impactor, most energy is rejected by the soft guiding structure, which is made possible by structural bending of the rods. After the impact, the guiding structure completely recovers to its original position for reusability. Such a regenerative feature of the 1D curved hybrid chain differentiates from plastic deformation or buckling behavior of conventional shock mitigation structures.

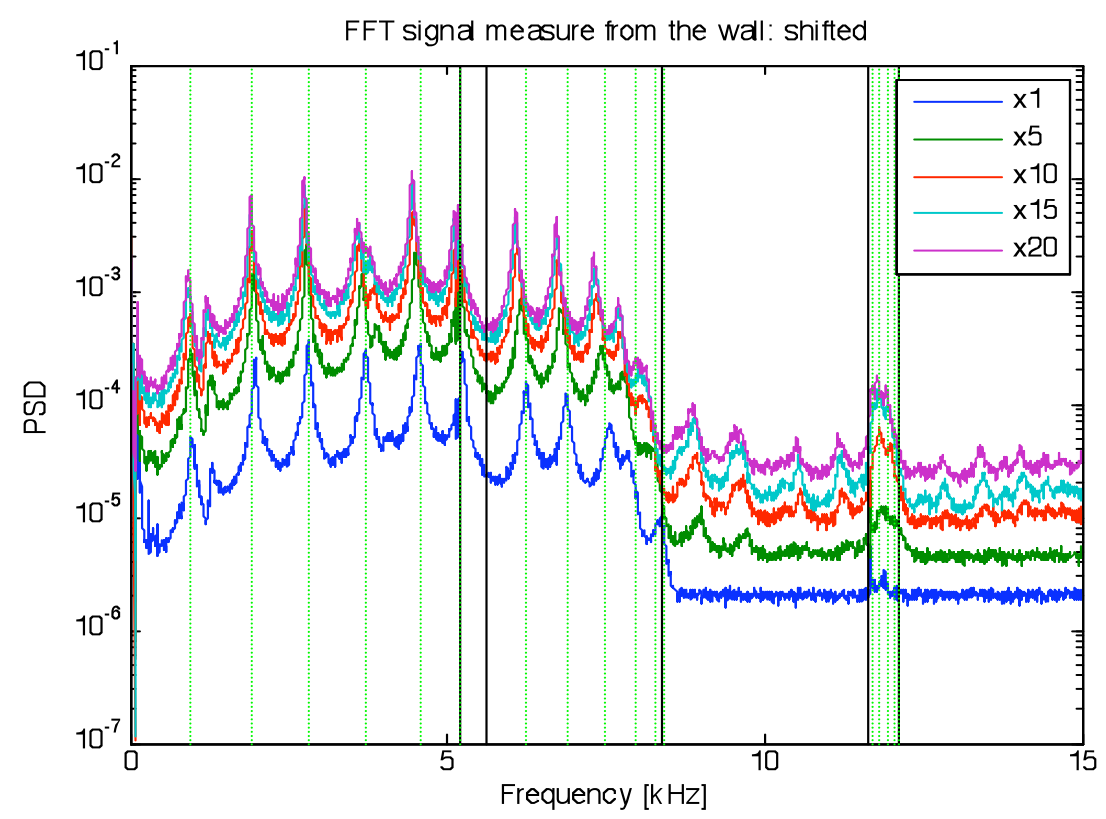

Figure 4. Frequency band structures of a diatomic granular chain under various amplifier gains. Harmonic excitations are applied to an end of the chain, and the transmitted waves are measured on the other side of the chain using a force sensor. Black vertical lines denote theoretical cutoff frequencies, while green dotted lines represent analytic resonant frequencies of the finite-length granular structure.

\section{Experimental results}

In experiment, we found that this curved hybrid structure is extremely efficient in absorbing high impact energy, compared to the 1D straight granular chain. We performed experiments by colliding the 1D curved chain with various cylindrical strikers with their heights varying from $1 / 4$ " to 24 ". The diameters of the stainless steel strikers were kept constant at 3/4". The force profiles measured from the force sensor are shown in Fig. 6. Under the low impact with light masses, the hybrid chain supports the propagation of a single solitary wave for the efficient transmission of impact energy [see the bottom blue curve in Fig. 6]. If the chain is impacted with a heavy striker, we observe the generation of shock-like waves [7], which decay fast due to the shock mitigation by the dispersive and structurally deformable linear guides [see the top gray force profile in Fig. 6]. Further experimental results will be presented in the later sections. 


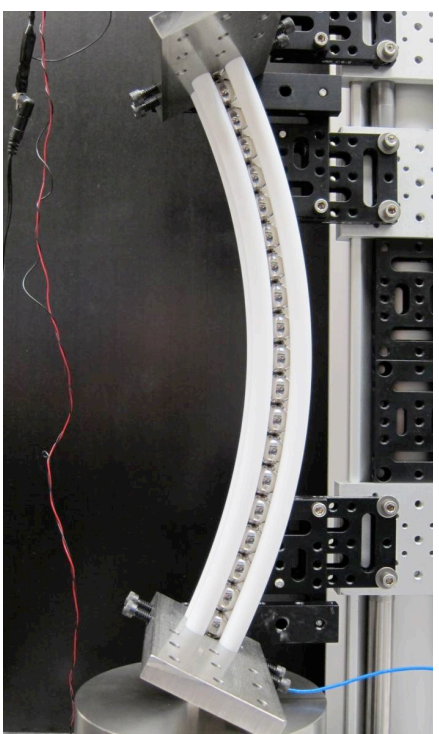

Figure 5. 1D curved chain composed of 20 stainless steel beads guided by four pre-curved PTFE cylinders.

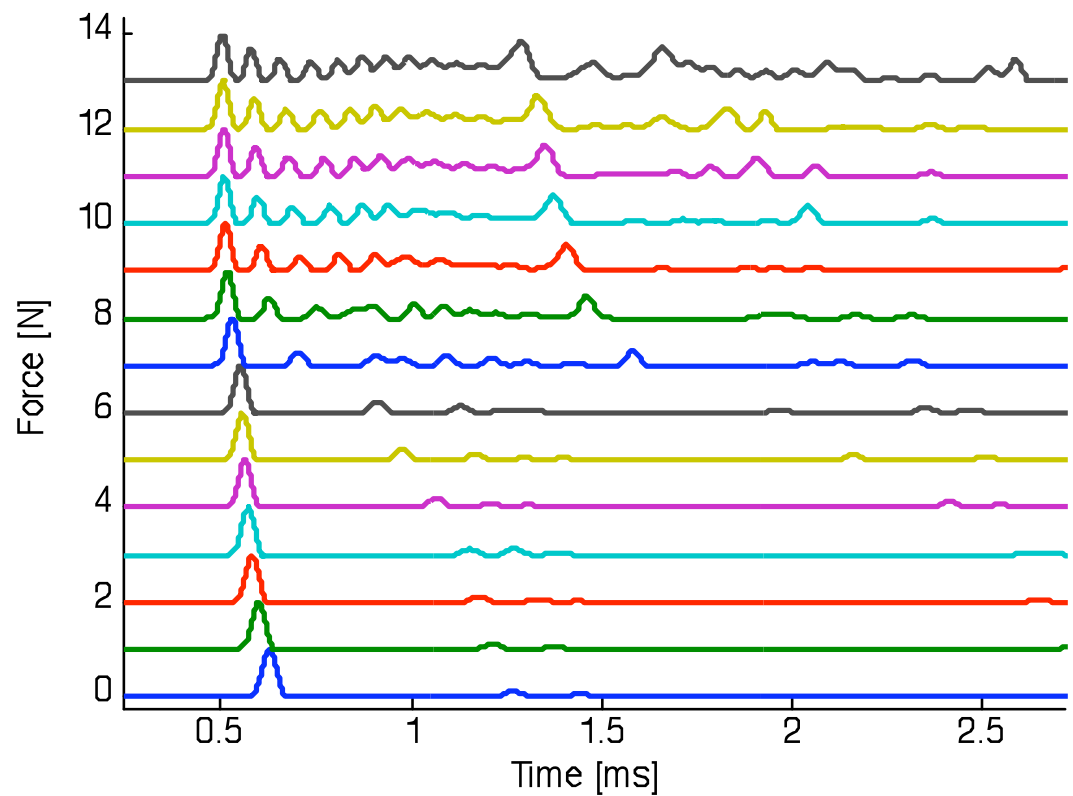

Figure 6. Experimental force profiles measured from the force sensor located at the bottom of the 1D curved hybrid chain. The chain is impacted by 14 different cylindrical strikers with 1/4" (bottom blue curve) to 24" (top gray curve) heights and constant 3/4" diameters.

\section{Numerical studies on transient responses of 1D curved chain}

\section{Numerical model}

To verify the experimental results, we developed an integrated discrete particle and finite element model. The discrete particle model is used to simulate the solitary wave propagation in the granular chain [2], while the finite element method using a 3-degree of freedom (DOF) beam element calculates the structural behavior of the PTFE guides under the interaction with the granular chain. The discrete particle model in the curved configuration needs a two-dimensional consideration of the particles' translational and rotational motion, which makes the problem more complex than that of the 1D chain as described in Eq. (1). The detailed equations of motion are described in the Appendix.

\section{Comparison of experimental and numerical results}

We compared the acoustic filtering performance of the curved hybrid chain with that of the straight uniform chain in both numerical and experimental terms. Figure 7 shows the force profiles of transmitted waves measured from the force sensor located at the base of the chain under three different impactors. Given small-amplitude impact, the force profiles and maximum magnitudes between the straight and curved chains are almost identical as depicted in blue curves of Figs 7 (a) and (b). However, under the shock-like impact caused by a heavier striker, it is observed that the curved chain develops a distinctive force profile compared to that of the straight chain. Specifically, the maximum amplitude of the shock front is significantly reduced, and the subsequent pulses of the shock also decay faster with high attenuation. This trend has been successfully reproduced by the numerical simulations as shown in Figs. 7 (c) and (d). The highly-attenuated force profiles in the strong impact cases are due to the shock mitigation by the soft guiding rails, which efficiently absorb the impact using spring-like structural behavior.

The aforementioned findings in the temporal-domain signals can be confirmed in the surface plots that collectively show the transmitted force profiles in various impact conditions [Fig. 8]. We observe that both the straight and the curve chains well transmit the impact energy in the form of solitary waves under the small-mass impact collision, which are represented by a single hump of highlighted zone 
in the lower parts of the graphs in Figs. 8 (a) and (b). However, as the impact amplitude increases, the shock-like waves start to be formed and the curved chain obviously dissipates the energy more efficiently than the straight chain. We can verify this by reduced intensity of the force amplitude in the curved chain configuration, as shown in upper highlighted zone of Fig. 8 (b). We confirm this experimental finding by the numerical results [Figs. 8 (c) and (d)].
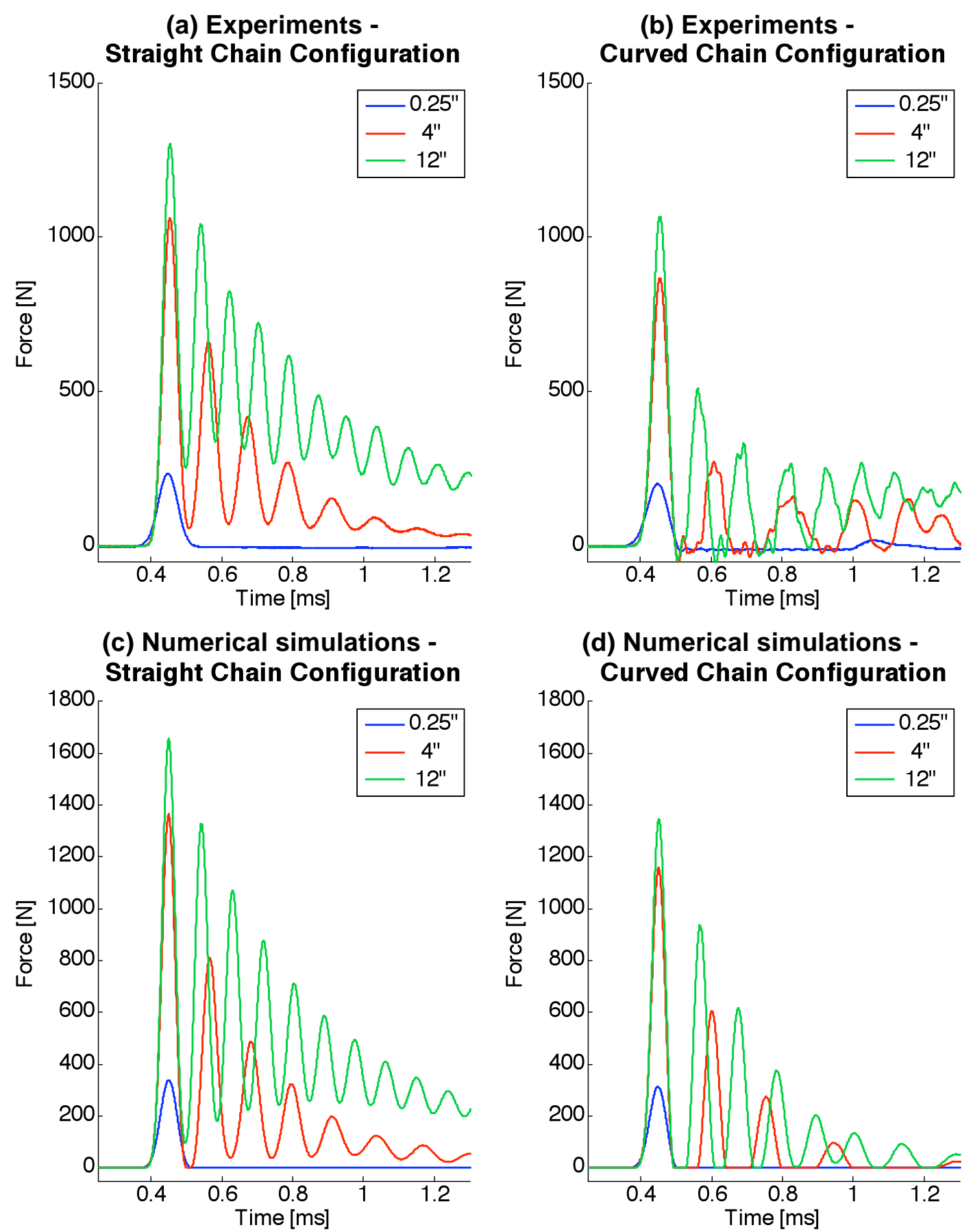

Figure 7. Experimental and numerical results of the force profiles under different striker masses for the straight and the curved chains. Using a small-mass impactor, almost identical force profiles in the shape of a single solitary wave are obtained for both straight and curve chain configurations. However, as the mass increases, shock-like responses are witnessed with distinctive force profiles between the straight and the curved chains. We can observe that the curved chain dissipates the shock more efficiently with smaller peak amplitude and faster decay. 


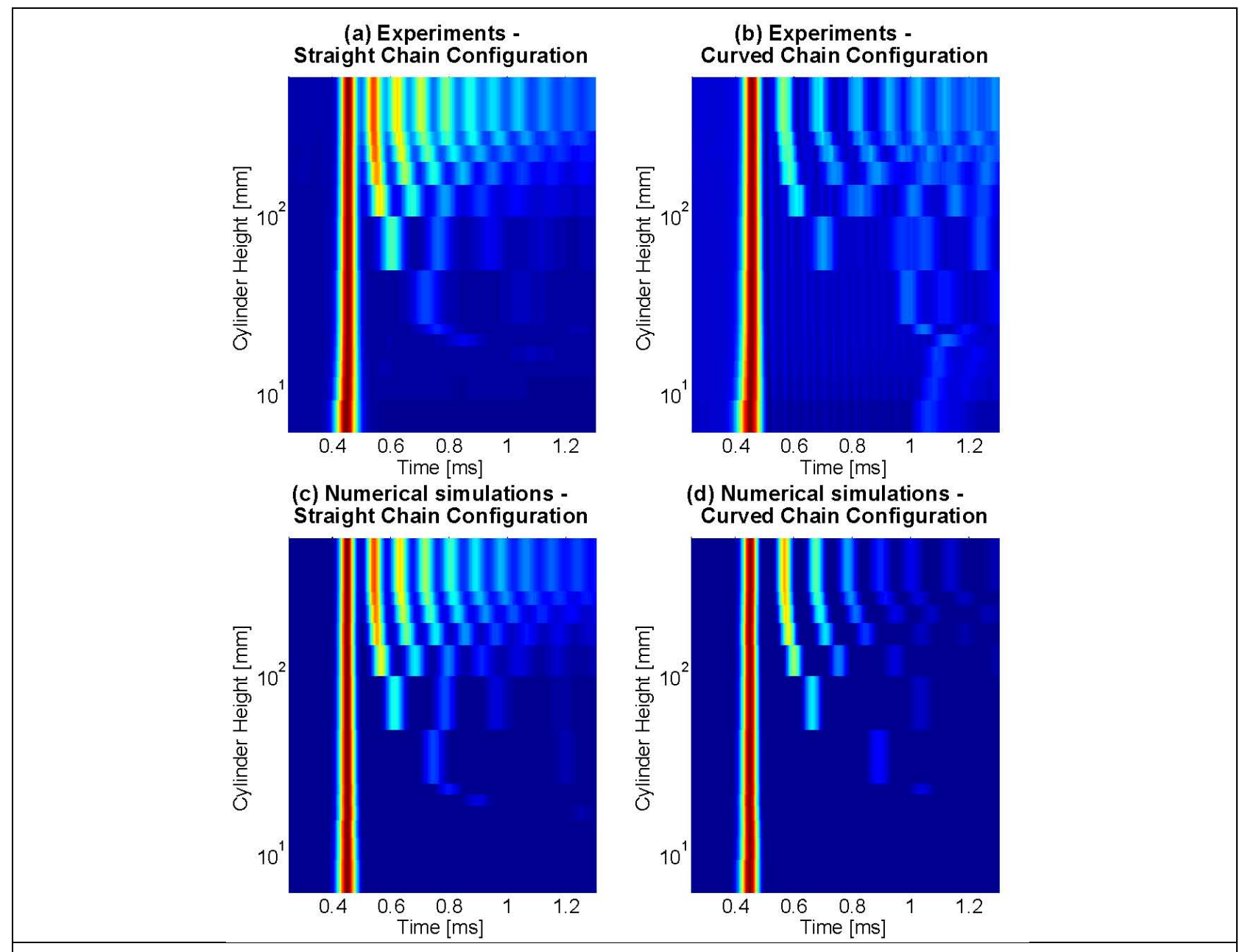

Figure 8. Experimental and numerical surface plots of transmitted waves for straight and curved chain configurations. Fourteen cylindrical strikers with different thickness from 1/4" to 24" are used to impose various impact amplitudes. For the lower amplitude impacts, a single hump of solitary waves is formed. On the other hand, a train of shock-like waves is observed in the case of the larger-mass impacts. The discrepancy between the straight and curved chain responses is obvious in the strong impact case, where faster decay of shock is observed in the curved chain configuration.

\section{Comparison of filtering performances between straight and curved uniform chains}

We quantified the energy carried by the transmitted waves by calculating the second norm energy (SNE) of the measured force profiles. The SNE values for straight and curved chain configurations are reported in Fig. 9 based on both experimental and numerical results. We find that the transmitted amounts of energy are almost equivalent under the small-mass impact for both chains. However, they start to deviate from each other as the impact amplitude increases by large-mass striker impacts. This means that the curved chain transmits less amounts of energy under strong impact compared to the straight chain. The experimental results are confirmed in the numerical simulations as shown in Fig. 9(b). Due to the possible onset of plasticity under the heavy striker impact, the SNE value exhibits a significantly decrease in experiments compared to numerical simulations [see the last point of the red curve in Fig. 9(a)].

The ratios of the curved chain's SNE values to those of the straight chain are shown in Fig. 10. According to the experimental results, we observe that the ratio is as high as over $80 \%$ for smallamplitude impact, whereas it is reduced to less than $20 \%$ under the strong impact. Numerical results predict slightly higher values than the experimental ones, due to the fact that the numerical model does not account for friction or plasticity effect. It is very interesting that we obtained a large variation of SNE 
ratios using the 1-D curved chain of hybrid linear/nonlinear structure. This implies that using this nonlinear acoustic metamaterial, we can achieve the intended performance of the amplitude-tunable acoustic filtering in a selected amplitude region.
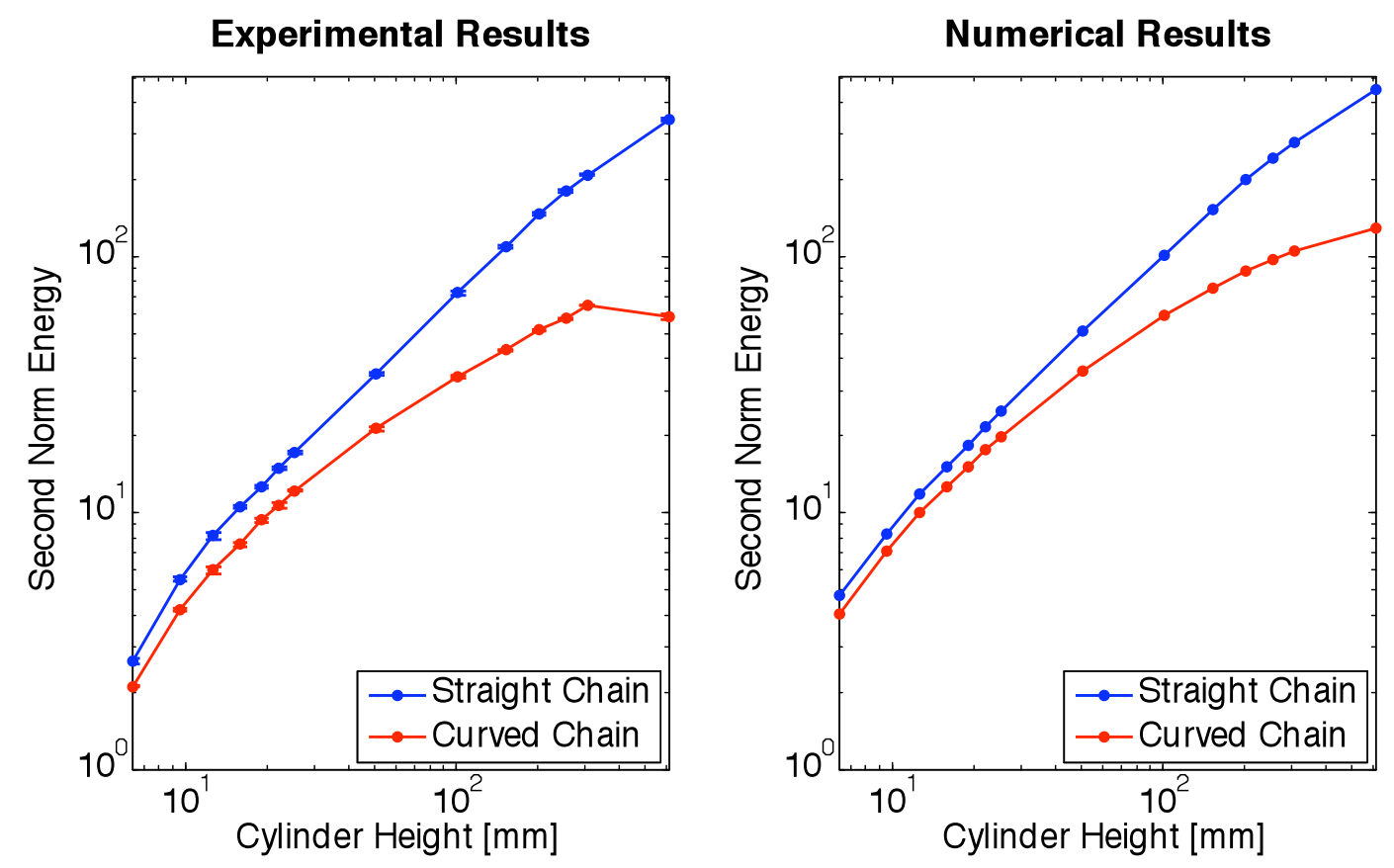

Figure 9. Experimental and numerical results of second norm energy (SNE) values in the straight and curved chain configurations. As heights (i.e., mass) of cylindrical strikers increase, the transmitted amounts of energy in the curved chain are relatively diminished in comparison to those of the straight chain.

\section{Testing of a diatomic curved chain under impacts and continuous excitations}

\section{Experimental Setup}

We assemble one-dimensional (1D) granular crystals composed of diatomic unit cells under the constraint of curved and straight elastic guides [Figs. 10(a) and (b)]. Each unit cell consists of "spherecylinder" particles made from stainless steel (type 440C, McMaster-Carr). The spherical elements have a radius $R_{\mathrm{s}}=9.53 \mathrm{~mm}$, mass $m_{\mathrm{s}}=28.2 \mathrm{~g}$, elastic modulus $E_{\mathrm{s}}=200 \mathrm{GPa}$, and Poisson's ratio $v_{\mathrm{s}}=0.28$. The cylindrical elements have a radius $R_{\mathrm{c}}=9.53 \mathrm{~mm}$, a height $h=9.53 \mathrm{~mm}$, and mass $m_{\mathrm{c}}=42.4 \mathrm{~g}$, with material properties identical to those of the spherical elements. The chains are 21 particles long with 10 repeating unit cells $(N=10)$ and one additional spherical particle $(i=21)$ at the bottom of the chain [see Fig. 11]. 

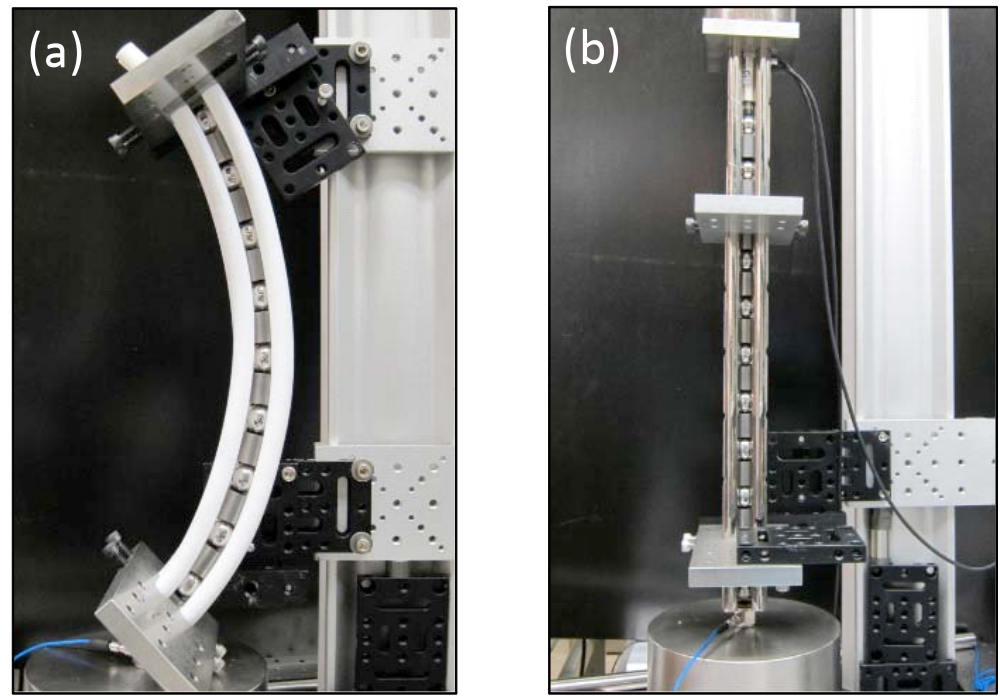

Fig. 10. Experimental setup. (a) Curved configuration of a diatomic chain composed of sphere-cylinder unit cells. Each unit cell comprises a sphere $\left(R_{s}=9.53 \mathrm{~mm}\right)$ and a cylinder $\left(R_{c}=9.53 \mathrm{~mm}, h_{c}=9.53 \mathrm{~mm}\right)$. The chain is 21 particle long (10 unite cells and one supplementary sphere at the top), and the particles are constrained by 4 PTFE tubes. (b) Straight configuration of identical particles confined by 4 stainless steel rods. The height of the straight chain is $0.40 \mathrm{~m}$.

The curved diatomic chains are guided by four polytetrafluoroethylene (PTFE) tubes with an outer radius $R_{G, o}=6.35 \mathrm{~mm}$, an inner radius $R_{G, i}=4.76 \mathrm{~mm}$, density $\rho_{G}=4302 \mathrm{~kg} / \mathrm{m}^{3}$, elastic modulus $E_{G}$ $=0.46 \mathrm{GPa}$, and Poisson's ratio $v_{G}=0.46$. Upon the external excitations applied on the top of the granular chains, these PTFE tubes allow flexible deformation due to their soft elastic property and light mass. The curvature of the chain is determined by the initially-bent PTFE tubes, which are held by the upper and lower stainless steel plates [Fig. 11]. We represent the curvature of the chain by $\Delta$, which measures the offset of the chain from its centerline. We test eight different curved chains $(\Delta=[22.4,26.0,35.2,45.1$, $58.9,66.2,73.3,85.2] \mathrm{mm})$. A customized linkage structure provides vertical and rotational degree of freedom of the upper plate, allowing the assembled chain structurally bent under external excitations. As a reference, we also consider a straight chain as a reference configuration. In this case, we restricted lateral motion of diatomic granular particles using hard stainless steel rods [Fig. 10(b)].

In this study, we apply two different types of external excitations to the granular chains: broadband disturbances and impulsive excitations. We use broadband disturbances to generate small amplitude excitations relative to those due to the static compression. This allows evaluating the dynamic responses of the diatomic chain under linear dispersion. To generate broadband disturbances, we use a commercial piezoelectric actuator (Physik Instrumente, P-212 piezostack actuator), powered by an external voltage amplifier [inset (a) of Fig. 11]. We condition its output with white-noise signals (1second duration) generated by MATLAB. The piezoelectric actuator is positioned on the top of the chain in a direct contact with the top sphere. This piezoelectric actuator is mounted to the support plate, which is guided vertically by four stainless steel rods. To impose precompression to the granular chain, we simply place a ring-shaped weight on the top of the support plate. In this study, the total precompression imposed by the weight and support plate is $56.4 \mathrm{~N}$. 

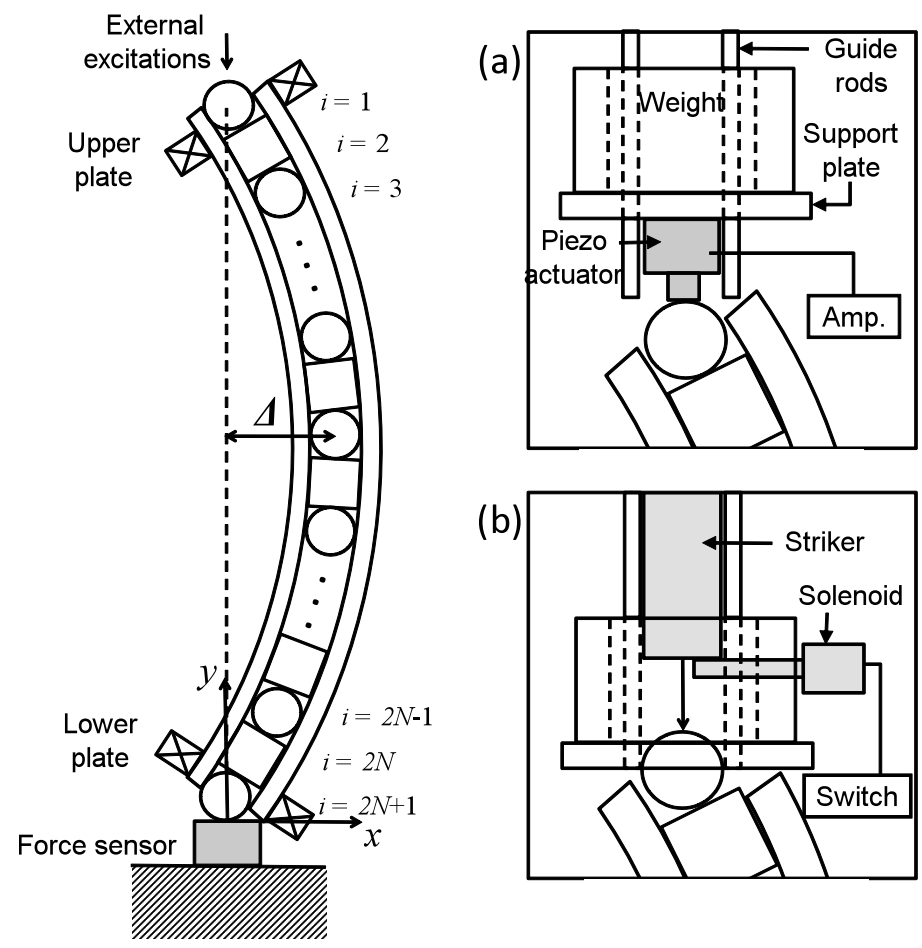

Fig. 11. Schematic of experimental setup. The curvature of the diatomic chain, represented by an offset $\Delta$ from the centerline (dashed line), is determined by initially bent PTFE guides, which are held by the upper and lower plates. The propagated waves through the chain are measured by the force sensor mounted at the base. Via direct contact with the top spherical particle, two different types of external excitations are applied to the diatomic chain: (a) Harmonic excitations using a piezoelectric actuator, which is aligned to the chain using a support plate and guiding rods. We apply precompression to the chain by placing a ring-shaped weight on the top of the support plate. (b) Striker impacts using cylindrical masses. We release the striker from a 10-mm drop height using a DC-powered linear solenoid. The diatomic chain is precompressed using a ring-shaped weight and a resting plate that partially exposes the top of the sphere for the striker impact.

The inset (b) of Fig. 11 illustrates the impulse-excitation configuration via striker impacts. Compared to the dynamic disturbances generated by a piezoelectric actuator, we can achieve orders of magnitude higher compressive force using striker impacts. Therefore, we can investigate the dynamics of the diatomic granular chains in weakly and even highly nonlinear regimes. To simulate various nonlinearity condition, we employ 13 strikers with a different mass $M=[14.1,21.2,28.2,35.3,42.3,49.4,56.5,113$, $226,339,452,565,678] \mathrm{g}$. The strikers are made of stainless steel cylinders with a 9.53-mm radius and length $L=[6.35,9.53,12.7,15.9,19.1,22.2,25.4,50.8,102,152,203,254,305] \mathrm{mm}$ for each of the corresponding cylinder masses. To impact the top sphere of the chain, we release strikers from a 10-mm drop height using a DC-powered solenoid [20]. We apply precompression to the chain by resting a weight on the top of the support plate, which exposes partially the top sphere through an open hole for striker impacts. The ring-shaped weight is cut open to allow access of the solenoid from the lateral direction [see the inset (b) of Fig. 11].

We measure the transmitted waves through the diatomic chain using a commercial force sensor (Piezotronics, PCB-C04 force sensor), mounted on a massive block that simulates a rigid wall. The physical stiffness of the force sensor is $1.05 \mathrm{kN} / \mu \mathrm{m}$, which is much higher than the contact stiffness between diatomic particles. The sensor is connected to a data acquisition board (National Instrument PCI$6115)$ to collect signals at a sampling frequency up to $10 \mathrm{MHz}$. The acquired signals are processed in MATLAB. For the statistical treatment of signals, we average the measurements over five acquisitions. 


\section{Numerical Approach}

Previous studies have demonstrated the presence of frequency band structures for 1D granular crystals, when their dynamic responses are within a linear regime [4, 25-27]. In particular, a diatomic granular chain has been shown to exhibit an acoustic transmission spectrum with evident pass- and stopbands. Under small-amplitude dynamic excitations relative to precompression, the linearized equations of particles' motion in our "cylinder-sphere" diatomic chain can lead theoretically to the following dispersive relation [27]:

$$
m_{s} m_{c} \omega^{4}-2 \beta\left(m_{s}+m_{c}\right) \omega^{4}+2 \beta^{2}(1-\cos (a k))=0,
$$

where $m_{s}$ and $m_{c}$ are the sphere's and cylinder's masses, $\omega$ is the angular frequency, $k$ is the wave number, and $a$ is the equilibrium length of the unit cell. The coefficient $\beta$ is derived from the linearized Hertzian contact relationship within the unit cell, which can be expressed as:

$$
\beta=\frac{3}{2} A^{2 / 3} F_{0}^{1 / 3} \text {, where } A=\frac{4 \sqrt{R}}{3}\left(\frac{1-v_{s}^{2}}{E_{s}}+\frac{1-v_{c}^{2}}{E_{c}}\right)^{-1} \text {. }
$$

Here $F_{0}$ denotes precompression initially applied to the granular crystal, and the subscripts $s$ and $c$ represent material properties of spheres and cylinders, respectively. In this equation, we assume spheres and cylinders have the identical radii $\left(R=R_{\mathrm{c}}=R_{\mathrm{c}}\right)$. If we solve the dispersion relation in Eq. (1) for $k=\frac{\pi}{a}$ and $k=0$, we obtain the following cutoff frequencies:

$$
\begin{aligned}
& f_{1}=0 \\
& f_{2}=\frac{1}{2 \pi} \sqrt{\frac{2 \beta}{m_{c}}} \\
& f_{3}=\frac{1}{2 \pi} \sqrt{\frac{2 \beta}{m_{s}}} \\
& f_{4}=\frac{1}{2 \pi} \sqrt{\frac{2 \beta\left(m_{s}+m_{c}\right)}{m_{s} m_{c}}} .
\end{aligned}
$$

The frequency bands in $\left[f_{1}, f_{2}\right]$ and $\left[f_{3}, f_{4}\right]$ are called acoustic and optical bands, respectively, that allow the transmission of acoustic waves through the granular crystals. On the other hand, the frequency band in $\left[f_{2}\right.$, $f_{3}$ ] prevents the propagation of acoustic bands, referred to as band-gap. The cutoff frequencies as expressed in Eq. (3) are dictated by material properties, geometry, and precompression applied to the granular crystals, allowing tunability of frequency band structures.

This theoretical validation of acoustic band spectrum is built on the 1D straight-chain configuration under the assumption of linear responses of the granular crystals. To investigate the transition of band structures in curved granular crystals, we use numerical approach that combines discrete element (DE) and finite element (FE) methods [28]. This combined method allows modeling both the particles dynamics and the kinematics of the support tubes, given various chain configurations and nonlinearity conditions. In previous studies, 1D granular crystals have been successfully modeled as chains of point-masses connected by nonlinear springs based on Hertzian contact interaction [1-3, 5-8]. In such discrete element models (DE) the particles are considered as rigid bodies and dissipative losses are neglected. It has been shown that the dynamics of short granular chains can be well represented without the need of fitting parameters $[2,3,6,10]$. The DE model, that includes two-dimensional interactions, 


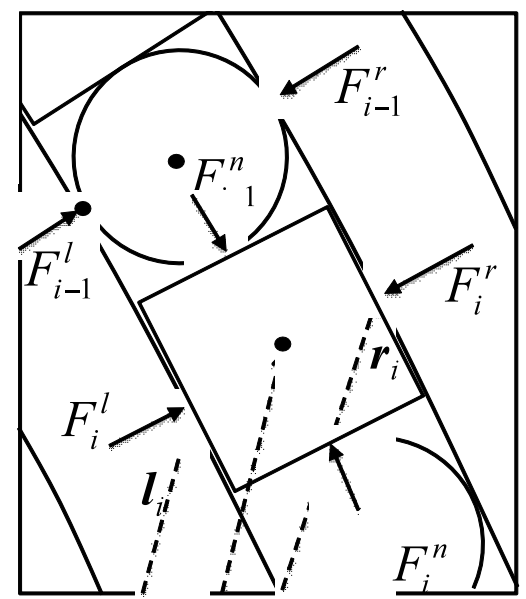

Fig. 12. Free body diagram of the sphere-cylinder unit cell under the normal forces exerted by neighboring particles and contacting guides. Here dotted arrows represent location vectors of contact points, while solid arrows denote force vectors.

captures the dynamics of the curved granular crystals. The FE model is used to analyze the dynamic behavior of the linear elastic guides, which are discretized into Bernoulli-Euler beam elements [29].

We first describe the equations of particles' motion in the DE model using Newtonian mechanics $[1,23]$. The schematic diagram of the granular particles confined by elastic guides is illustrated in Fig. 12. In this closely packed diatomic granular chain, the increment of relative normal approach between the $i$-th and $(i+1)$-th particles can be written as:

$$
\delta_{i, i+1}^{n}= \begin{cases}R-\left|\boldsymbol{s}_{i+1}-\boldsymbol{s}_{i}\right| & i=0 \\ R+h / 2-\left|\boldsymbol{s}_{i+1}-\boldsymbol{s}_{i}\right| & i \in\{1, \ldots, 2 N\} \\ R-\left|\boldsymbol{s}_{i}\right| & i=2 N+1\end{cases}
$$

Here $s_{i}$ is the location vector of the $i$-th particle's center with respect to the origin, which is the contact point between the last particle and the rigid wall [see the coordinate and its origin in Fig. 11]. The vector $\boldsymbol{s}_{0}$ indicates the piezoelectric actuator tip or the bottom center of striker masses, implying the external excitations imposed to the granular crystals. The number of unit cells $N$ is 10 in this study, and the last particle embedded between the granular chain and the force sensor is represented with index $i=21$.

Using Hertzian contact relationship, the nonlinear interaction between adjacent particles can be expressed [30]:

$$
F_{i, i+1}^{n}=A\left[\delta_{i, i+1}^{n}\right]^{/ 2}
$$

with Hertizan coefficient $A$ defined in Eq. (2). Here $F_{i, i+1}^{n}$ denotes normal (i.e., compressive) force between the $i$-th and $(i+1)$-th elements. The bracket $[x]_{+}$takes only positive values and returns 0 if $x<0$, implying a zero tensile strength among granules. This means that the DE model for the tightly-packed granular chain is governed by the double nonlinearity: a nonlinear force under compression and a zero tensile force [21].

The lateral supporting forces exerted on the sphere by the right- and left-hand linear guides, are regulated by the modified Hertzian contact of a sphere or a cylinder on a cylinder [31]. For the sake of brevity, the three-dimensional interplay of the guiding rails and the granular chain is simplified to twodimensional representation. The relative normal displacements between the $i$-th particle and the right- and left-hand guides are 


$$
\begin{aligned}
& \delta_{i}^{r}=R-\left|\boldsymbol{s}_{i}-\boldsymbol{r}_{i}\right| \\
& \delta_{i}^{l}=R-\left|\boldsymbol{s}_{i}-\boldsymbol{l}_{i}\right|,
\end{aligned},
$$

where $\boldsymbol{r}_{i}$ and $\boldsymbol{l}_{i}$ are the location vectors of the granular particle's contact with the right- and left-hand guides [Fig. 12]. In the case of a spherical element $(i \in\{1, \ldots, 2 N+1\})$, the Hertzian contact force between the $i$-th particle with its neighboring guides can be written as

$$
\begin{aligned}
& F_{i}^{r}=B_{s}\left[\delta_{i}^{r}\right]^{/ 2} \\
& F_{i}^{l}=B_{s}\left[\delta_{i}^{l}\right]^{/ 2} .
\end{aligned}
$$

The coefficient $B_{s}$ is expressed as $B_{s}=K_{s}\left(\frac{1-v_{s}^{2}}{E_{s}}+\frac{1-v_{G}^{2}}{E_{G}}\right)^{-1}$ where subscript $s$ and $G$ denote the material properties of the sphere and the guiding tubes, respectively. The coefficient $K_{s}$ is determined by the geometry of the rod and sphere, based on the analytical expression of Hertzian contact between a sphere and a cylinder [31]. Given the test configuration, we use $K=0.105$ in this study.

If a cylindrical element develops a contact with the elastic guides, we assume a line contact between a cylinder and the elastic guides and apply two dimensional contact law between them [31]. Unlike the Hertzian contact law in Eqs. (4) and (6), the contact relationship between the cylindrical particle $(i \in\{2, \ldots, 2 N\})$ and the right-hand elastic guide is described in a complicated mathematical expression as below:

$$
\delta_{i}^{r}=F_{i}^{r}\left(V_{c}+V_{G}\right)\left[1+\ln \left\{\frac{h^{2}}{F_{i}^{r}\left(V_{c}+V_{G}\right)}\left(\frac{1}{R_{c}}+\frac{1}{R_{G}}\right)\right\}\right]
$$

where $V_{s}=\frac{1-v_{s}{ }^{2}}{E_{s}}$ and $V_{G}=\frac{1-v_{G}{ }^{2}}{E_{G}}$. The expression for the contact with the left-hand guide is identical to Eq. (7) with the replacement of superscript from $r$ to $l$. Based on this equation, our numerical model accounts for the interaction between the cylindrical members in the diatomic chain and the bent guides that surround them.

In our DE model, we neglect the friction between the granules and the guiding structure due to the relatively small normal forces and smooth surface of PTFE. Furthermore, we do not consider rotational friction between spherical and cylindrical particles, based on previous reports showing that the amount of energy dissipated through slip or rotational friction at the contact is much less than the energy accumulated by normal contact forces in confined force chains [32]. Thus, taking all the normal force components into account, the acceleration of the $i$-th particle can be determined by the relation

$$
\boldsymbol{a}_{i}=\frac{\sum \boldsymbol{F}_{i}}{m_{i}}+\boldsymbol{g}=\frac{\boldsymbol{F}_{i-1, i}^{n}+\boldsymbol{F}_{i, i+1}^{n}+\boldsymbol{F}_{i}^{r}+\boldsymbol{F}_{i}^{l}}{m_{i}}+\boldsymbol{g}
$$

The superscript $n$ denotes the axial interaction with neighboring particles, while $r$ and $l$ represent the normal interaction with the left- and right-hand beam elements [see Fig. 12]. We include gravity $g$ in the consideration of the weight of granular particles.

We use a single array of beam finite elements (FE) to model the curved guides. Each element has two nodes with three degrees of freedom in axial, shear, and rotational directions. The governing 
equations of motion for the right- and left-hand guiding structures can be expressed in a linear algebraic form as:

$$
\begin{aligned}
& {\left[\boldsymbol{M}^{r}\right] \ddot{\boldsymbol{r}}=\left[\boldsymbol{K}^{r}\right] \dot{\boldsymbol{r}}+\boldsymbol{F}^{r}} \\
& {\left[\boldsymbol{M}^{l}\right] \ddot{\boldsymbol{l}}=\left[\boldsymbol{K}^{l}\right] \dot{\boldsymbol{l}}+\boldsymbol{F}^{l},}
\end{aligned}
$$

where $\boldsymbol{r}=\left[r_{1, x}, r_{1, y}, \cdots, r_{N, x}, r_{N, y}\right]^{T}$ and $\boldsymbol{l}=\left[l_{1, x}, l_{1, y}, \cdots, l_{N, x}, l_{N, y}\right]^{T}$ with subscripts $x$ and $y$ denoting $x$ - and $y$-directional vector components. The matrices $\left[\boldsymbol{M}^{r}\right]$ and $\left[\boldsymbol{K}^{r}\right]$ are the global mass and stiffness matrices of the right-hand elastic guide, while $\left[\boldsymbol{M}^{l}\right]$ and $\left[\boldsymbol{K}^{l}\right]$ are for the left-hand elastic guide.

Based on equilibrium, the vectors $\boldsymbol{F}^{r}$ and $\boldsymbol{F}^{l}$ that represent the exerted force on the right and left supports by the granular chain are expressed as

$$
\begin{aligned}
\boldsymbol{F}^{r} & =\left[F_{1, x}^{r}, F_{1, y}^{r}, \cdots, F_{N, x}^{r}, F_{N, y}^{r}\right]^{T} \\
\boldsymbol{F}^{l} & =\left[F_{1, x}^{l}, F_{1, y}^{l}, \cdots, F_{N, x}^{l}, F_{N, y}^{l}\right]^{T} .
\end{aligned}
$$

This force vector connects the equations of motion for the discrete granular elements in Eq. (7) with those for linear guides in Eq. (9). The ordinary differential equations for both the nonlinear granular chain and the linear medium are solved with the explicit Runge-Kutta integration scheme in MATLAB [33].

\section{TECHNICAL RESULTS}

\section{IV-1. Broadband Excitations}

We first investigate the effect of curvature on the linear dispersive response of granular crystals. We analyze the frequency band structure of curved granular crystals based on the compressive waves transmitted through the granular crystals and measured at the base of the chain. In experiments, we observe the magnitude of the measured force profiles is within $\pm 0.5 \mathrm{~N}$. This ensures the dynamic disturbances imposed by the external excitations are orders of magnitude smaller than the static precompression $\left[F_{0}=56.4 \mathrm{~N}, \operatorname{Order}\left(F_{\mathrm{d}} / F_{0}\right) \approx 10^{-2}\right]$. For numerical simulations, we generate white-noise signals that have maximum force disturbance in the order of $10^{-1}$, compared to the static precompression of the diatomic chain. Such small ratios of dynamic perturbations in comparison to the static precompression allow the dispersive wave propagation of elastic waves within the granular crystals.

Figure 13 reports the frequency spectra of acoustic waves transmitted through diatomic chains with four different curvatures. The vertical lines represent analytic predictions of cutoff frequencies from Eq. (3). Given the precompression and diatomic chain configurations, we obtain $f_{2}=6.46 \mathrm{kHz}, f_{3}=7.91$ $\mathrm{kHz}$, and $f_{4}=10.2 \mathrm{kHz}$. From the experimental results in Fig. 13 (a), we observe the presence of both acoustic and optical bands, that include resonant frequencies of finite diatomic chains denoted by sharp spikes. Under zero offset, i.e., in the straight chain, the pass bands are within the analytic cutoff frequencies. As the chain curvature increases, however, we find the frequency bands are down-shifting significantly. In particular, the optical pass band is positioned in the region of analytic band-gap, when the chain curvature becomes $85.2 \mathrm{~mm}$. The numerical results in Fig. 13 (b) corroborate the experimental results, showing that the transmission of the compressive waves through the curved chains is dependent on the initial curvature imposed to the system.

We include surface plots of the experimental and numerical results in Fig. 14, to show the transmitted power spectrum density (PSD) for various curvatures imposed on the diatomic chains. We use diatomic chains of eight different curvatures $(\Delta=[0,22.4,35.2,58.9,66.2,73.3,85.2] \mathrm{mm})$ for experimental testing, while we evaluate the linear dispersion of transmitted waves for every 5-mm offset 
from $\Delta=0 \mathrm{~mm}$ to $100 \mathrm{~mm}$ in a numerical setting. Here the dashed yellow lines denote the analytic cutoff frequencies of acoustic and optical pass bands. The color bars show the PSD scale, representing the ratio of transmitted wave amplitudes to the input signal amplitudes (also called as transfer function gain). In both experimental and numerical results, the upper bright band corresponds to the optical pass band, while the lower bright band denotes the acoustic pass band. The presence of a stop band between the optical and acoustic bands is evident in Figs. 14 (a) and (b).
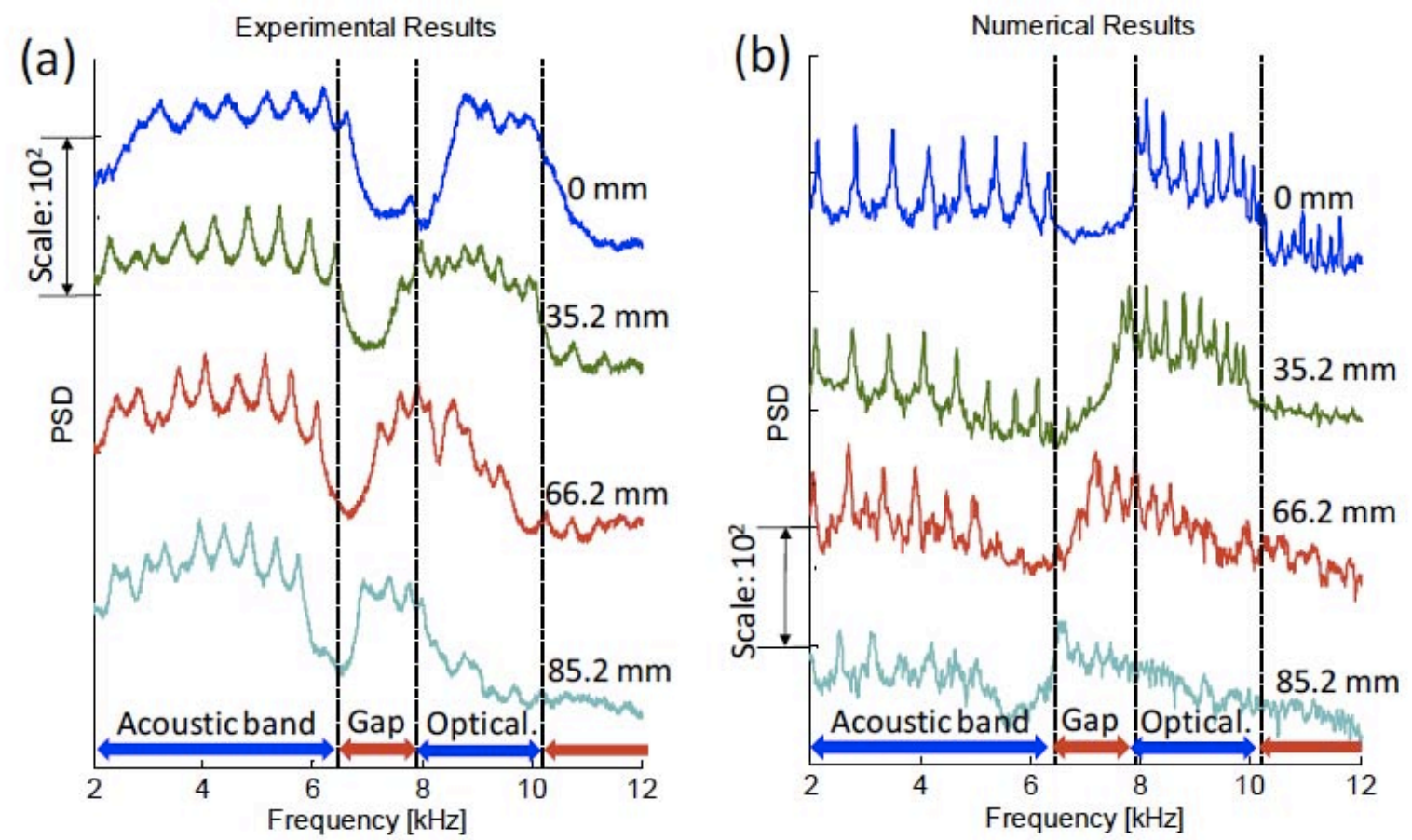

Figure 13. Power spectral density (PSD) of transmitted waves for the curved chain with $\Delta=[0,35.2,66.2,85.2]$ $\mathrm{mm}$. To ease visual comparison, the signals obtained from the different curvatures are shifted vertically along the logarithmic Y-axis, where each grid represents a $10^{2}$ gain. (a) Experimental results. As the curvature of the diatomic chain increases, the frequency band structure, particularly optical pass band, is shifted towards a lower frequency domain. (b) Numerical results. Despite the increased noise level, the presence of allowable and forbidden bands and the effect of chain curvature on the frequency shift are evident.
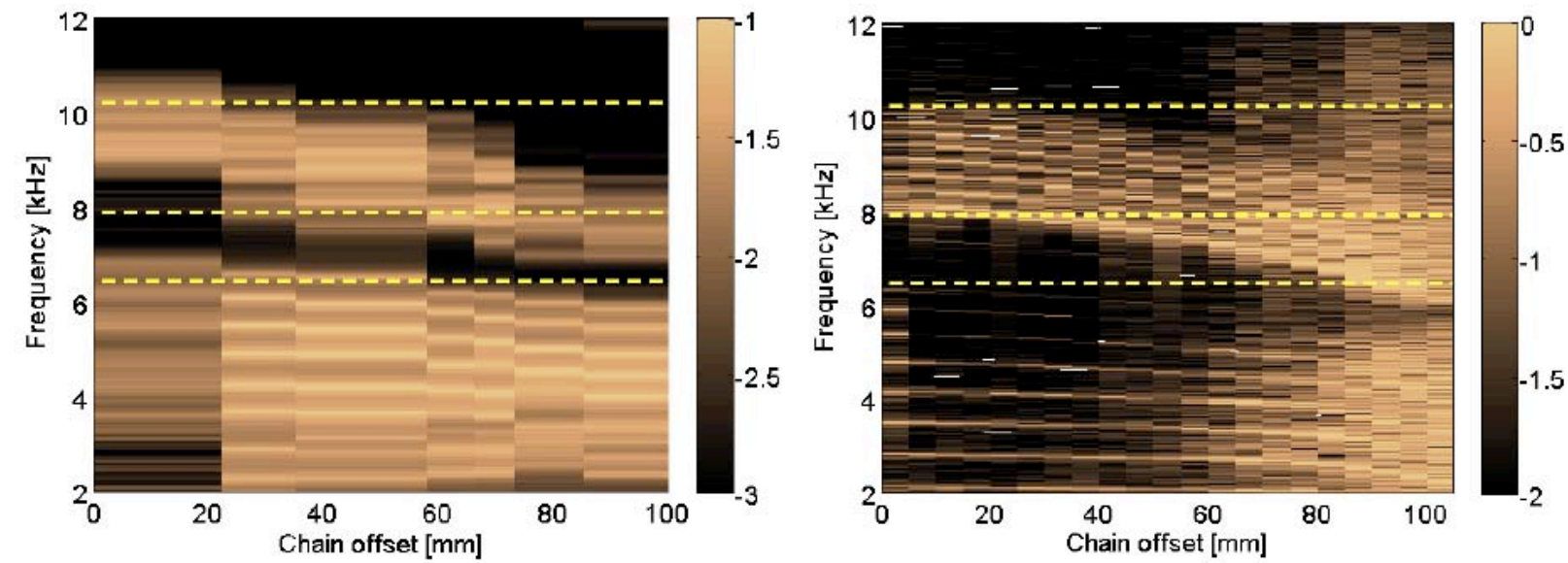

Figure 14. Surface maps of PSD as a function of diatomic chain curvature. (a) Experimental results for $\Delta=[0,22.4$, $35.2,58.9,66.2,73.3,85.2] \mathrm{mm}$. (b) Numerical results for the various curvatures. The color bar reports the ratio of transmitted-wave amplitudes to those of input excitations. Dashed yellow lines denote cutoff frequencies of acoustic and optical pass bands. 
From the surface plots, we can confirm the effect of chain curvature on the acoustic transmission spectrum. We find that the frequency bands are shifting towards a lower frequency zone, as the curved chain exhibits a larger curvature. In particular, the shift of the optical pass band is more drastic than the acoustic band. As a result, the optical band is repositioned nearly in the region of analytic band-gap at large curvatures around $\Delta=80 \mathrm{~mm}$ for both experiments and simulations. Hence, besides precompression, geometry, and material properties of granules, we find that curved granular crystals provide an additional means to control acoustic band structures by simple manipulation of chain curvatures.

In the experimental results of a straight chain, we find the frequency bands are up-shifted compared to the analytic frequency bands predicted by Eq. (3) [see the upper pass band on the leftmost side of Fig. 14 (a)]. Such upshift in the frequency spectrum at an approximately 5\% range has been previously observed through experiments of granular crystals, and possible explanations have been discussed in [34]. Compared to the experimental results, numerical calculations of acoustic transmission spectrum are in an excellent agreement with analytic predictions for the straight chain [see the highlighted zone in the leftmost side of Fig. 14 (b)]. Also we find the PSD values are larger than the experimental results [compare the color bars of Figs. 14 (a) and (b)]. This is because the combined DE and SE method do not take dissipation effect into account, while the presence of significant attenuation of transmitted waves is evident in experiments. The discrepancy of relative PSD intensity of optical and acoustic band passes between the experimental and numerical results is probably due to the dissipation effect as well.

\section{IV-2. Impulse Excitations}

In this section, we characterize the dynamic responses of the bent granular crystal structure in the weakly- and highly-nonlinear regime by imposing a wide range of impacts using different strikers. We begin investigating the propagation of waves upon the light mass impact $(M=14.1 \mathrm{~g})$ with a velocity of $0.443 \mathrm{~m} / \mathrm{s}$. The temporal force profiles recorded by the sensors positioned at the end of the chain are reported in Fig. 15 (a) for the straight chain (solid blue line) and curved chain (dashed red line). Here the initial offset $\Delta$ of the curved chain is $58.9 \mathrm{~mm}$. For the lightest striker, which has a mass smaller than that of a bead ( $m=28.2 \mathrm{~g}$ ), we find that both the straight and the curved chains result in the formation of an impulsive wave followed by the oscillatory waves. The maximum amplitude of the compressive wave is in the order of precompression applied to the structure $\left[\operatorname{Order}\left(F_{\mathrm{d}} / F_{0}\right) \approx 1\right]$, ensuring the excitation of the structure in the weakly-nonlinear regime. Hence, the wave formed in the chain does not exhibit the shape of highly nonlinear solitary waves in a diatomic chain. Comparing the responses of the straight and curved chains, we observe that the wave triggered by the light striker in the curved chain is very similar to that in the straight chain. However, the maximum force is reduced by $22.9 \%$ as compared to the straight chain (from $104 \mathrm{~N}$ in the straight chain to $80.2 \mathrm{~N}$ in the curved one). This is due to losses of the axial force components in the support rods.

We then characterize the wave propagation upon the largest mass impact $(M=678 \mathrm{~g})$. In Fig. 15(b), we observe that the maximum force induced by the striker $\left(F_{d}=\sim 1,000 \mathrm{~N}\right)$ is much larger than the precompression $\left(F_{0}=56.4 \mathrm{~N}\right)$, implying the enhanced degree of nonlinearity compared to the previous weakly-nonlinear condition. Unlike the light mass impact, we observe the generation of trains of solitary waves, particularly for the straight chain, which are characterized by a leading pulse with large amplitude and trailing waves with amplitudes decaying approximately exponentially [22]. Another notable difference is that the chain of solitary waves excited in the straight and curved chains present a remarkably different behavior, when the heaviest striker impacts the chain. Comparing the decay patterns of the train of waves, we find that the transmitted waves in the curved chain results in faster decay than that in the straight chain. The maximum amplitude of the compressive force is also reduced by $40.3 \%$, from $1027 \mathrm{~N}$ in the straight chain to $613 \mathrm{~N}$ in the curved one [see Fig. 15(b)]. Overall, the area under the force-time curve, which corresponds to the amount of momentum transferred, is significantly reduced in the curved chain compared to that of the straight one, implying that the curved systems can attenuate large-amplitude signals more effectively. Numerical results in Figs. 15(c) and 15(d) are found to agree well with the experimental findings. 
The frequency spectra of transmitted waves are shown in Fig. 16 for various strikers. Both numerical and experimental results are based on the fast Fourier transformation of time-domain signals,

(a)

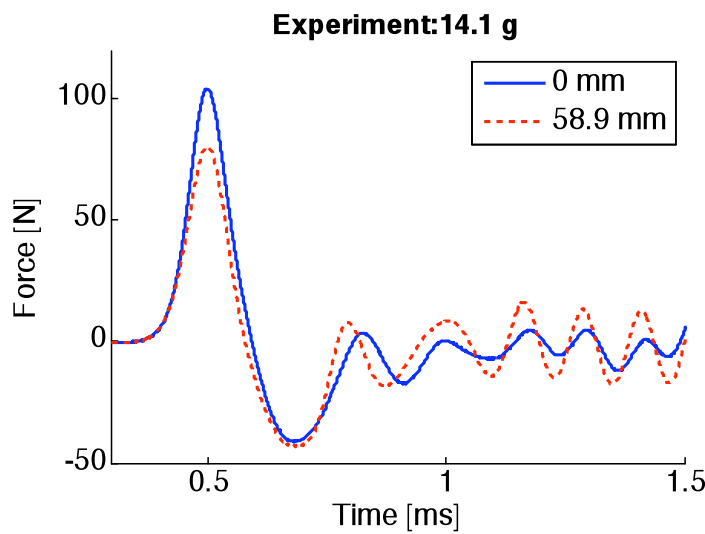

(c)

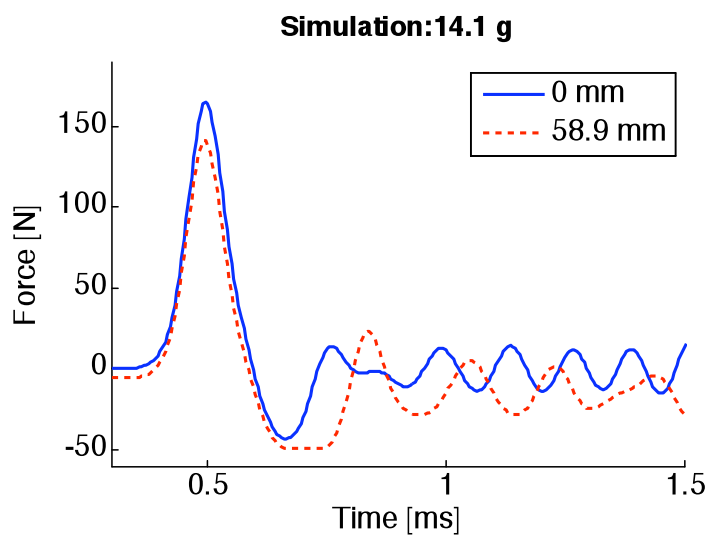

(b)

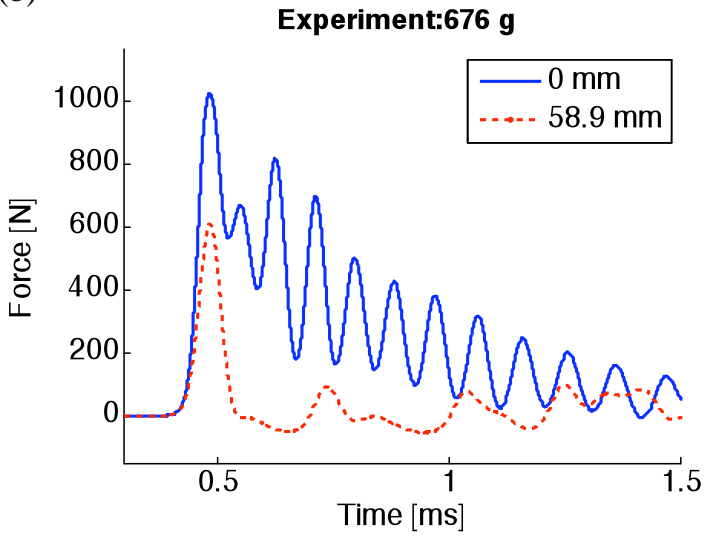

(d)

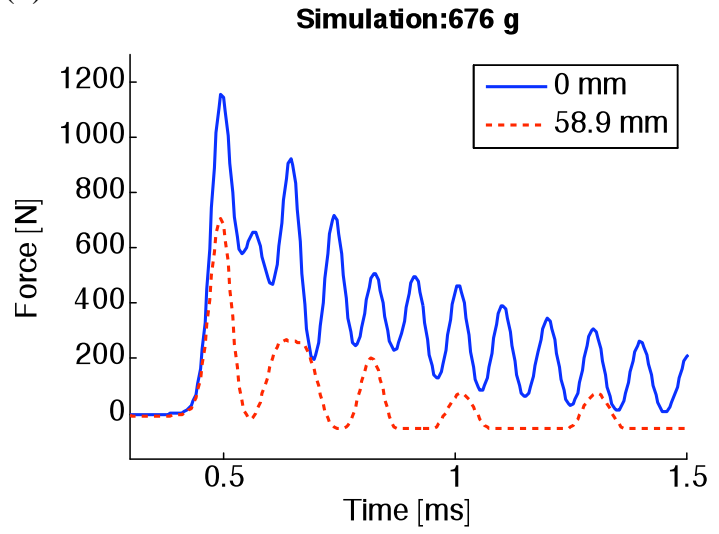

Figure 15. Experimental (top row) and numerical (bottom row) results of the force-time profiles for the light-mass (14.1g, left column) and the heavy-mass (678g, right column) impacts. Here, the transmitted force profiles are measured by the force sensor located at the base of the granular chains. For the straight chain, the impact of the light mass generates a dynamic impulse followed by oscillatory waves, while the heavy-mass impact forms a train of waves that decay approximately exponentially. For the curved chain with an initial curvature $\Delta=58.9 \mathrm{~mm}$, a similar pattern of compressive wave propagation is observed for the light-mass impact. However, for the heavy-mass impact, we witness a significant attenuation of transmitted waves with smaller initial amplitudes and faster decay, compared to the compressive waves transmitted along the straight chain.

which are normalized with respect to their maximum power spectral density (PSD) values. For the straight chain, we observe the presence of the optical and acoustic pass bands in the low range of striker masses [see Fig. 16(a)]. However, as the striker mass increases, the distinction between pass- and stopbands become less evident, and particularly, the intensity of the optical pass band decreases drastically. This means that weakly nonlinear waves are affected by dispersion mechanism, exhibiting acoustic transmission band structures as predicted by linear dispersion relationship. The propagation of highly nonlinear waves, however, does not show any presence of acoustic band structures. Figure 16(b) shows the frequency responses of the curved chain $(\Delta=58.9 \mathrm{~mm})$ under various impacts. We again confirm the dispersive effect of propagating waves under the light-striker impacts. In comparison to the straight chain, the band structure is down-shifted, and this is in agreement with the findings in the linear regime (see IV1. Broadband Excitations). The numerical simulations in Figs. 16 (c) and (d) corroborate the experimental results satisfactorily, though the existence of band-gap is not clearly seen in the curved chain. 


\section{IV-3. Energy Transmission}

We estimate the energy arriving on the wall by calculating the electrical energy stored in the piezoelectric sensor located at the base of the chain. The integral form of electrical energy $E_{\mathrm{C}}$ of the

(a)

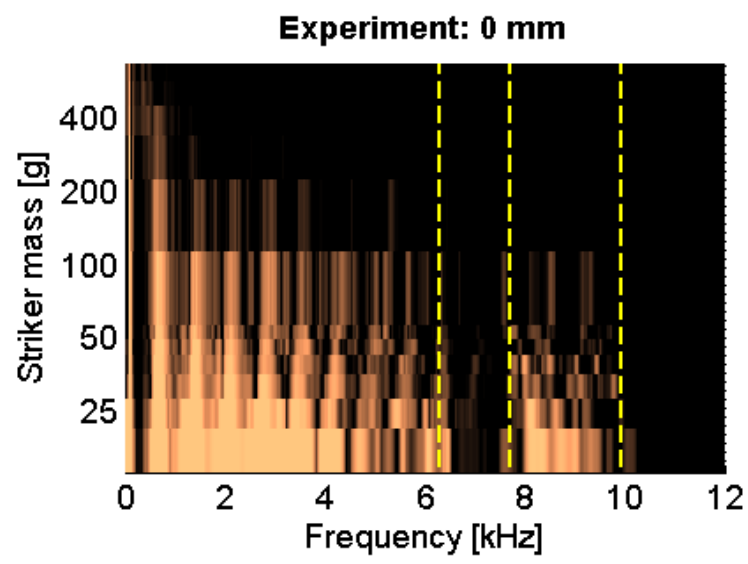

(c)

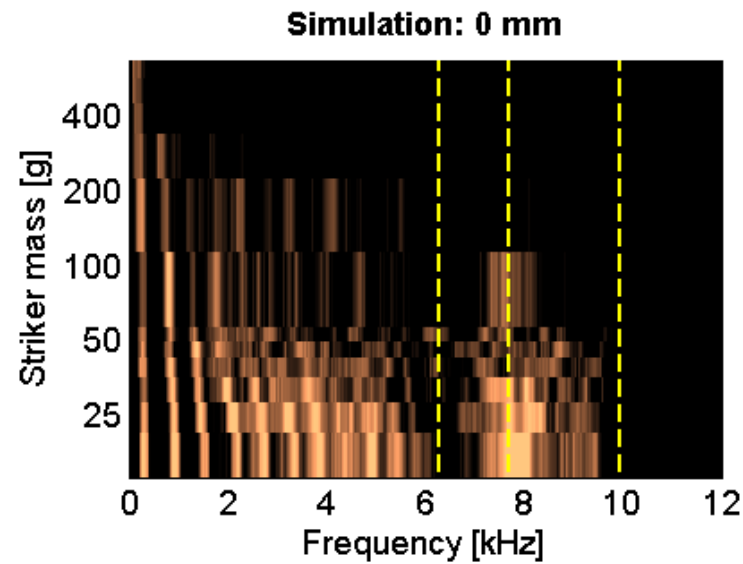

(b)

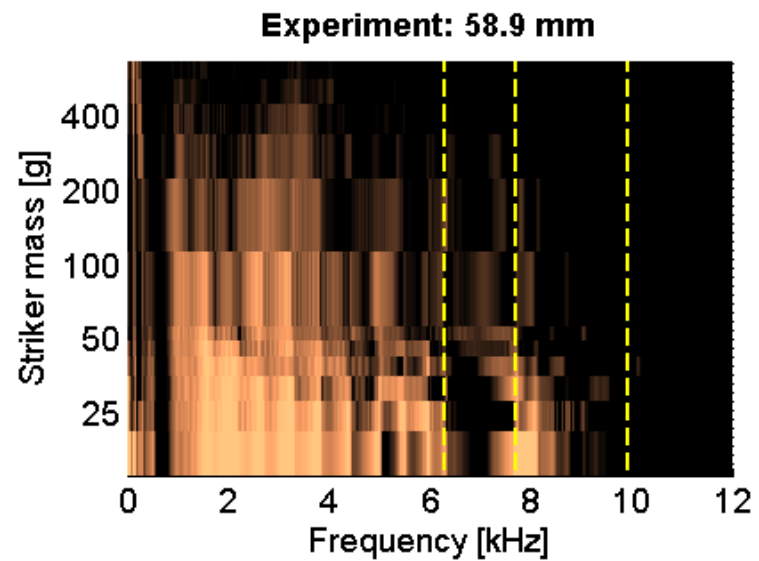

(d)

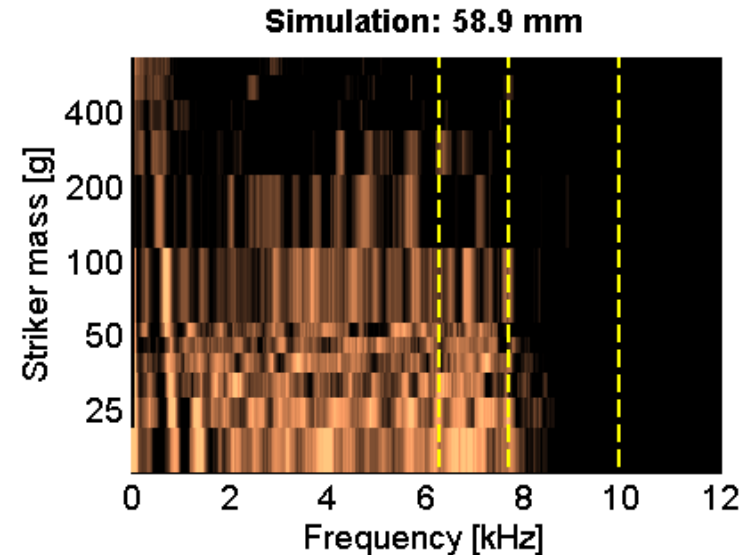

Figure 16. Experimental (top row) and numerical (bottom row) surface plots of PSD for straight (left column) and curved (right column) chains. Each surface plot depicts the force profiles of all 13 striker impacts after being normalized with respect to their maximum PSD values. The dashed yellow lines denote the band edges of acoustic and optical pass bands predicted by analysis. Under light-striker impacts, the band structure is observed with evident dependence on the curvature. However, as the striker masses increase, PSD in the optical band becomes extinct. transducer in the time domain of $\left[t_{i}, t_{f}\right]$ can be expressed as [35]:

$$
E_{G}=\int_{i=t_{i}}^{t_{f}} C \cdot V(t)^{2} d t,
$$

where $C$ is the capacitance of the transducer, and $V(t)$ is a continuous form of the voltage generated in the transducer. Assuming an approximately linear relationship between $V(t)$ and the applied force on the sensor in the force range considered [36], the discrete form of electrical energy in Eq. (11) can be simplified to the second norm of the discrete force profile $F[t]$ as follows: 


$$
S N E=\frac{1}{f_{s}} \sum_{t=t_{i}}^{t_{f}} F[t]^{2}
$$

where $f_{\mathrm{s}}$ is the sampling frequency of the transducer. In this study, we refer to this pseudo-energy as the second norm energy (SNE).

We compare the energy transmission through the chains as a function of the striker masses for four different chain curvatures $(\Delta=0,26.0,45.1$, and $58.9 \mathrm{~mm})$. Experimental results are shown in Fig. 17(a) and the corresponding numerical data is reported in Fig. 17(b). From these plots it is evident that the SNE transmitted through the straight chain is almost linear (blue line with open circles), implying that in the straight configuration the transmitted waves carry the impact energy without significant dissipation [1, 5]. The energy transmitted through the curved chains instead shows a clear dependence on the striker mass: as the striker mass is increased the energy transmitted is reduced compared to that in the straight configuration. Notably the amount of energy transmitted can be reduced by imposing a larger initial curvature to the chains. This allows tuning the amount of energy losses into the guides in the form of strain and kinetic energy. The numerical results are found in good agreement with the experimental data.
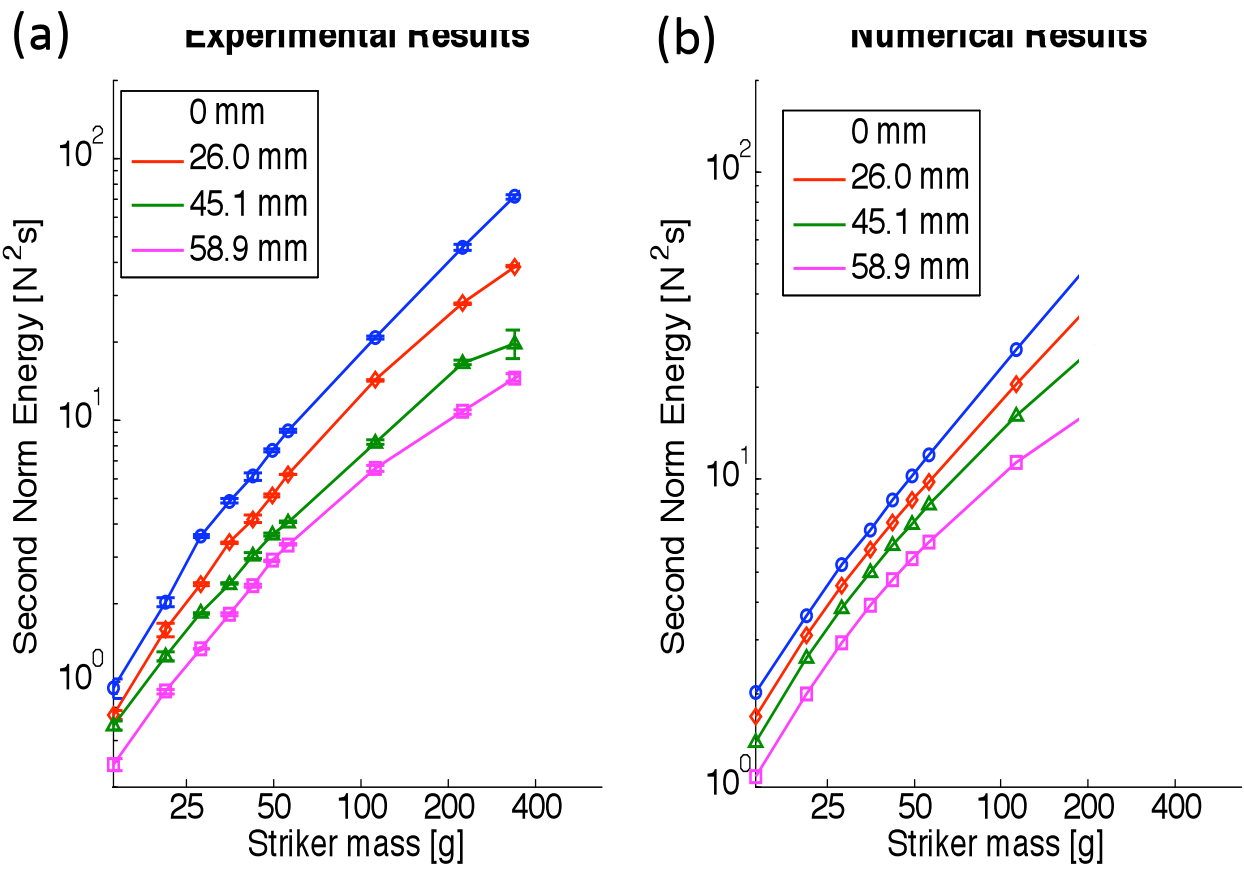

Figure 17. Experimental (left) and numerical (right) results of second norm energy (SNE) values as a function of striker masses. The chain configurations tested include a straight chain $(\Delta=0 \mathrm{~mm})$ and three curved chains $(\Delta=$ $26.0,45.1,58.9 \mathrm{~mm})$. The error bars in the experimental results denote standard deviations from five measurements of SNE values per chain curvature and striker mass. 


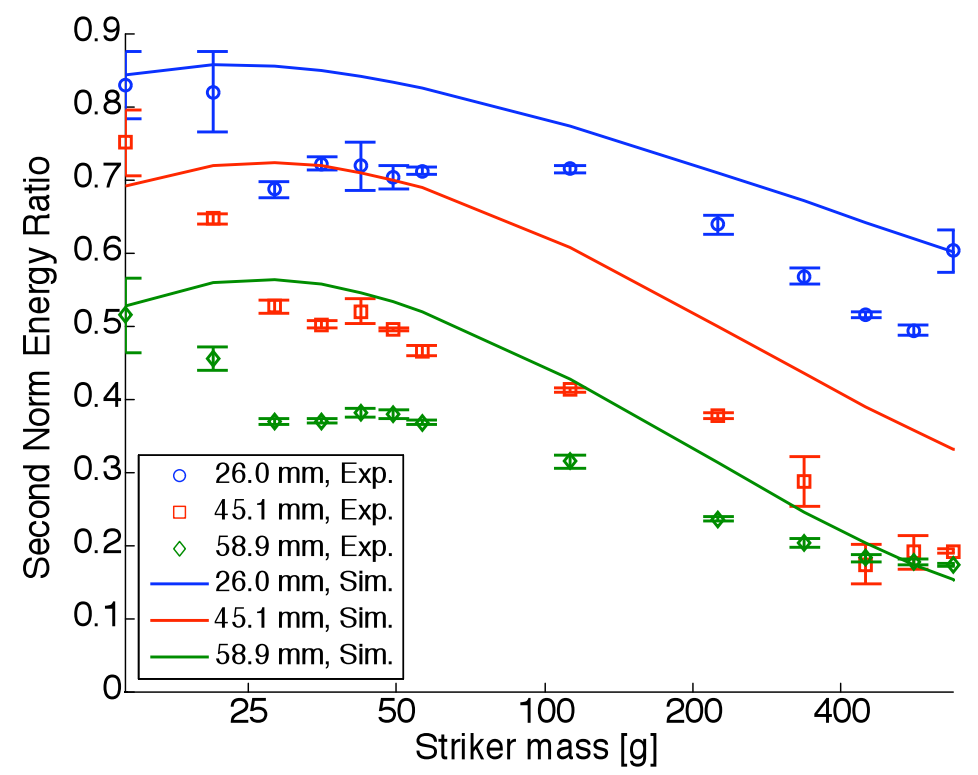

Figure 18. The ratios of the curved chain's SNE values to those of the straight chain as a function of striker masses. The solid lines represent numerical results, while the discrete points with error bars denote experimental results. As striker masses increase, the SNE ratios decrease accordingly, showing amplitude-dependent energy transmitting properties. A more drastic reduction of the SNE ratios is obtained for the granular chain with larger curvatures.

We quantify the energy transmission efficiency of the curved chains with respect to that of the straight chain in Fig. 18. Here, the experimental and numerical ratios of transmitted energy are plotted as a function of the different striker masses used for the impacts. From experiments, we find that a curved chain exhibits high transmission efficiency (close to 85\%) under small disturbances, while the efficiency drops as low as $18 \%$ when the chains are impacted with larger strikers. The experimental and numerical results are in fair agreement. We also observe that the experimental SNE ratios lie below the numerical ones for larger disturbances. This is probably due to the increased effects of friction and viscoelasticity when the system is excited with larger strikers. It is important to mention that no visible onset of permanent deformation in the particles or structural buckling is observed in the range of impacts tested in this study. This implies the amplitude-dependent transmission of compressive waves can be achieved in a regenerative manner, using the combined system of granular crystals and linear elastic media.

\section{IMPORTANT FINDINGS AND CONCLUSIONS}

We construct a one-dimensional granular crystal composed of tightly packed "sphere-cylinder" diatomic particles. The granular crystal is constrained by initially bent guides made of soft PTFE rods. We impose a various range of external excitations to the granular crystals, encompassing linear to nonlinear regimes, and study the propagation of compressive waves through the granular crystal. As a result, we obtain the following important findings:

\section{(1) Experimental observation of tunable frequency band structures}

We observe the presence of acoustic and optical pass bands, when we excite the diatomic granular crystals in the linear and weakly-nonlinear regimes. Furthermore, we find that the wave transmission efficiency can be tuned by the manipulation of the curvature imposed on the granular crystal.

\section{(2) Amplitude-dependent attenuation of compressive waves}

We experimentally demonstrate that the transmission of compressive waves through the granular chain is dependent on the amplitude of external impact, resulting from the close interplay between the granular 
crystal and the linear elastic guides. The energy transmission efficiency is also a function of the curvature imposed on the combined system of diatomic granular crystals and soft linear elastic media.

\section{(3) Coupling of linear and nonlinear media}

We numerically and experimentally observe that the energy carried by nonlinear granular crystals is dispensed to the surrounding media via the interaction between linear and nonlinear media. We model such coupling based on an approach that combines discrete element (DE) and finite element (FE) methods. This model successfully simulates the frequency- and amplitude-dependent behavior of acoustic filter systems over the range of linear to highly nonlinear regimes.

Our experimental setup shows a frequency range of the band-gap around $6 \sim 8 \mathrm{kHz}$, which can be adjusted to lower frequency bands by controlling the pre-curvature of the system. The location of the band-gap can be also easily changed by using different materials and geometry of unit-cell particles, or imposing different precompression to the curved chain. Using the combination of these design parameters, we expect to build nonlinear acoustic filter systems that can cover a broader range of frequencies.

\section{EXTENSION TO 3-D SYSTEMS:}

\section{SIGNIFICANT HARDWARE DEVELOPMENT}

In this study, we demonstrate a 1D prototype of nonlinear acoustic material system for frequencyand amplitude-dependent filtering of external impacts. The developed system is composed of diatomic particles constrained by soft elastic guides [Fig. 19 (a)]. Under small-amplitude disturbances, the combined linear and nonlinear system transmits the elastic waves or sound waves through its granular chain with high efficiency. These elastic waves are affected by the linear dispersive relationship of diatomic granular chains, presenting allowable and forbidden frequency bands. This frequency spectrum can be tuned by the curvature initially imposed to the linear elastic guides. As a result, this structure exhibits tunable, frequency-dependent filtering capability. Upon the excitation by large-amplitude impacts, this hybrid linear and nonlinear structure can mitigate external impacts efficiently by dispensing the input energy to the soft elastic guides. This soft structure undergoes structural deformation under impact, so that the impact energy is converted to the strain and kinetic energy.

The mechanism developed in this study can be extended to 3D architecture by arraying the granular crystals in the 3D space of soft media. We design a 3D prototype of nonlinear acoustic filter systems using a 5-by-5 array of helical granular crystals [Fig. 19 (b)]. In this structure, each helical chain can be composed of diatomic spherical particles with a 4.76- $\mathrm{mm}$ diameter. Given the identical geometry of diatomic particles, we can obtain the wider band-gap in the acoustic transmission structure by using materials with more distinctive mechanical properties. For example, we can obtain a frequency band-gap in the range between 3.38 and $8.62 \mathrm{kHz}$ by assembling the diatomic chain made of stainless steel and PTFE particles according to the numerical simulations (pre-compression $=1 \mathrm{~N}$ ). In this case, the optical pass band is formed between 8.62 and $9.26 \mathrm{kHz}$. This also implies that an audible frequency range can be successfully targeted by relatively small unit cell particles. We expect that the frequency bands of this structure can be tuned by altering the number of rotations per unit length of the helical structure.

We prototype a 3D architecture of the acoustic metamaterial [Fig. 10(c)]. We use a 3D printer (Objet Connex 500, www.objet.com) to rapid prototype the acoustic metamaterial. For the matrix material, we use a rubber-like, TangoBlack material [37], to function as a soft linear medium. We fill the helical holes with diatomic unit cells made of stainless steel and PTFE spheres with a diameter of $4.76 \mathrm{~mm}$. A full experimental and numerical investigation of these three-dimensional systems will remain as the nextstage of this project in the future. 
(a)

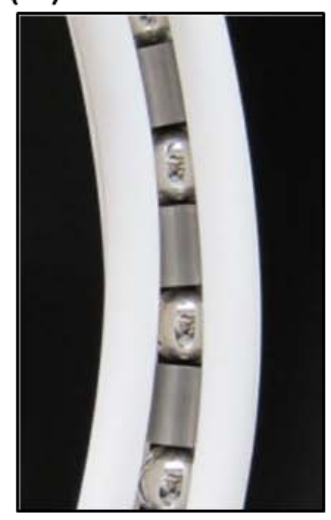

(b)

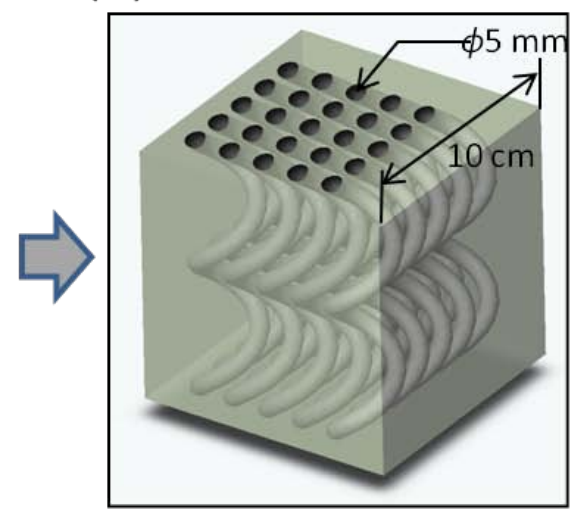

(c)

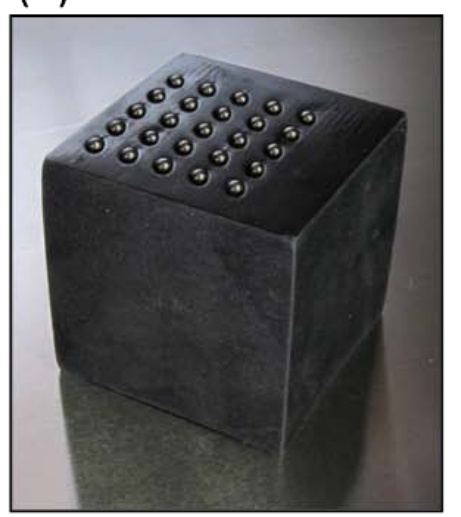

Fig. 19. (a) Diatomic "sphere-cylinder" chain constrained by soft PTFE guides. (b) 3D architecture of helical granular chains embedded in a soft medium. (c) 3D prototype made by 3D printer.

\section{IMPLICATIONS FOR FUTURE RESEARCH}

The findings of this work suggest the use of hybrid linear-nonlinear systems in engineering applications, such as tunable protective materials that selectively allow or forbid the transmission of external impacts, and novel acoustic devices for filtering and redirecting waves and mitigating vibrations. The characteristics of this new structure, made from nonlinear media held in a continuum matrix, can be tailored by varying material types, geometry, and structural arrangements of the composing elements. In particular, by including materials of contrasting properties (e.g., soft vs. rigid, elastic vs. plastic, solid vs. hollow), this structure becomes capable of presenting unique combinations of mechanical properties unprecedented in other mechanical systems. This tunable and lightweight materials system can form innovative structures used in space, civil infrastructure, and even biomedical applications, such as high pressure fuel tanks, next-generation energy storage systems, and new biomedical materials.

\section{REFERENCES}

[1] V. F. Nesterenko, Dynamics of Heterogeneous Materials, Springer-Verlag New York, Inc, New York, 2001.

[2] C. Coste, E. Falcon, S. Fauve, Physical Review E 56 (1997) 6104-6117.

[3] C. Daraio, V. F. Nesterenko, E. B. Herbold, S. Jin, Physical Review Letters 96 (2006) 058002.

[4] A. C. Hladky-Hennion, M. de Billy, J Acoust Soc Am 122 (2007) 2594-2600.

[5] S. Sen, J. Hong, J. Bang, E. Avalos, R. Doney, Physics Reports 462 (2008) 21-66.

[6] C. Daraio, V. F. Nesterenko, E. B. Herbold, S. Jin, Phys Rev E Stat Nonlin Soft Matter Phys 73 (2006) 026610.

[7] N. Boechler, J. Yang, G. Theocharis, P. G. Kevrekidis, C. Daraio, Journal of Applied Physics (2011), in print.

[8] M. Manciu, S. Sen, A. J. Hurd, Physical Review E 63 (2001) 016614.

[9] A. Spadoni, C. Daraio, Proc Natl Acad Sci U S A (2010) 7230-7234.

[10] C. Daraio, V. F. Nesterenko, E. B. Herbold, S. Jin, Physical Review E 72 (2005) 016603.

[11] J. B. Hong, A. G. Xu, Applied Physics Letters 81 (2002) 4868-4870.

[12] D. R. Khatri, P.; Daraio, C., Highly Nonlinear Waves' Sensor Technology for Highway Infrastructures, in: SPIE Smart Structures/NDE, 15th annual international symposium, San Diego, CA, 2008, pp. Manuscript number 6934-6925. 
[13] G. Theocharis, M. Kavousanakis, P. G. Kevrekidis, C. Daraio, M. A. Porter, I. G. Kevrekidis, Phys Rev E Stat Nonlin Soft Matter Phys 80 (2009) 066601.

[14] C. Daraio, V. F. Nesterenko, Shock Compression of Condensed Matter - 2007, Pts 1 and 2955 (2007) 1419-1422.

[15] C. Daraio, D. Ngo, V. F. Nesterenko, F. Fraternali, Physical Review E 82 (2010) 036603.

[16] R. L. Doney, S. Sen, Physical Review E 72 (2005).

[17] S. Job, F. Melo, A. Sokolow, S. Sen, Physical Review Letters 94 (2005).

[18] A. Rosas, A. H. Romero, V. F. Nesterenko, K. Lindenberg, Physical Review Letters 98 (2007) 164301.

[19] R. Carretero-González, D. Khatri, M. A. Porter, P. G. Kevrekidis, C. Daraio, Physical Review Letters 102 (2009) 024102.

[20] J. Yang, C. Silvestro, D. Khatri, L. De Nardo, C. Daraio, Physical Review E (2011), accepted.

[21] V. F. Nesterenko, C. Daraio, E. B. Herbold, S. Jin, Physical Review Letters 95 (2005) 158702.

[22] S. Job, F. Melo, A. Sokolow, S. Sen, Granular Matter 10 (2007) 13-20.

[23] A. Shukla, M. H. Sadd, Y. Xu, Q. M. Tai, Journal of the Mechanics and Physics of Solids 41 (1993) 1795-1808.

[24] A. F. Vakakis (Ed.) Normal Modes and Localization in Nonlinear Systems, John Wiley and Sons, New York, 1996, p.

[25] N. D. Boechler, C. , An experimental investigation of acoustic band gaps and localization in granular elastic chains in: Proceedings of the 22nd Biennial Conference on Mechanical Vibration and Noise VIB-5: Dynamics of Band-Gap Materials and Structures,, San Diego, CA, USA., 2009.

[26] E. Herbold, J. Kim, V. Nesterenko, S. Wang, C. Daraio, Acta Mechanica 205 (2009) 85-103.

[27] E. B. Herbold, J. Kim, V. F. Nesterenko, S. Wang, C. Daraio, Acta Mechanica 205 (2009) 18.

[28] J. Yang, S. Dunatunga, C. Daraio, (2011).

[29] P. I. Kattan, MATLAB guide to finite elements : an interactive approach, Springer, New York, 2007.

[30] K. L. Johnson, Contact Mechanics, in: Cambridge University Press, 1985.

[31] M. J. Puttock, E. G. Thwaite, National Standards Laboratory Technical Paper No. 25, Commonwealth Scientific and Industrial Research Organization, Australia (1969).

[32] A. Tordesillas, M. Muthuswamy, Journal of the Mechanics and Physics of Solids 57 (2009) 706-727.

[33] L. F. Shampine, M. W. Reichelt, SIAM J. Sci. Comput. 18 (1997) 1-22.

[34] N. Boechler, G. Theocharis, S. Job, P. G. Kevrekidis, M. A. Porter, C. Daraio, Physical Review Letters 104 (2010) 244302.

[35] J. Yang, F.-K. Chang, Smart Materials and Structures 15 (2006) 581.

[36] Piezoelectric Ceramics: Principles and Applications, APC International, Ltd., 2002.

[37] http://www.objet.com/3D-Printing-Materials/Overview/Tango_Materials/ 